\title{
Permanence and Almost Periodic Solution for an Enterprise Cluster Model Based on Ecology Theory with Feedback Controls on Time Scales
}

\author{
Yuanhong Zhi, Zunling Ding, and Yongkun Li \\ Department of Mathematics, Yunnan University, Kunming, Yunnan 650091, China \\ Correspondence should be addressed to Yongkun Li; yklie@ynu.edu.cn
}

Received 5 May 2013; Revised 2 September 2013; Accepted 2 September 2013

Academic Editor: Josef Diblík

Copyright (C) 2013 Yuanhong Zhi et al. This is an open access article distributed under the Creative Commons Attribution License, which permits unrestricted use, distribution, and reproduction in any medium, provided the original work is properly cited.

\begin{abstract}
We present a model with feedback controls based on ecology theory, which effectively describes the competition and cooperation of enterprise cluster in real economic environments. Applying the comparison theorem of dynamic equations on time scales and constructing a suitable Lyapunov functional, sufficient conditions which guarantee the permanence and the existence of uniformly asymptotically stable almost periodic solution of the system are obtained.
\end{abstract}

\section{Introduction}

In recent years, a few researchers have presented some models about enterprise clusters based on ecology theory, which arouse growing interest in applying the methods of ecology and dynamic system theory to study enterprise clusters, for example [1-9] and references cited therein. In [1], two models from biology were given and explained by economic view, and sufficient conditions were obtained to guarantee the coexistence and stability of enterprise clusters. In [3], the developing strategy of enterprise clusters was analyzed based on the logistic model, and the suggestions of constructing cooperative relation and choosing generalization or specialization tactics for commodity were put forward. In addition, based on the theoretical model of ecological population science, Wang and Pan [6] made a detailed analysis to the equilibrium mechanism of enterprise clusters, including net model and center halfback model and drew a conclusion that the relationship of pierce competition and beneficial cooperation among enterprise clusters was the crucial factor for them to keep stability. More related research about enterprises cluster one can refer to the literatures [10-13].
Recently, the literature [5] considered the competition and cooperation system of two enterprises based on ecosystem:

$$
\begin{aligned}
& \frac{\mathrm{d} x_{1}(t)}{\mathrm{d} t}=r_{1} x_{1}(t)\left(1-\frac{x_{1}(t)}{K}-\frac{\alpha\left(x_{2}(t)-c_{2}\right)^{2}}{K}\right), \\
& \frac{\mathrm{d} x_{2}(t)}{\mathrm{d} t}=r_{2} x_{2}(t)\left(1-\frac{x_{2}(t)}{K}-\frac{\alpha\left(x_{1}(t)-c_{1}\right)^{2}}{K}\right),
\end{aligned}
$$

where $x_{1}(t), x_{2}(t)$ represent the output of enterprises $A$ and $B, r_{1}, r_{2}$ are the intrinsic growth rate, $K$ denotes the carrying capacity of market under nature unlimited conditions, $\alpha$, $\beta$ are the competitive coefficients of two enterprises, $c_{1}, c_{2}$ are the initial production of two enterprises. Accordingly, we consider now the equation with nonconstant coefficients, which can be obtained as a modified system (1) with variable coefficients (Letting $a_{1}=r_{1} / K, a_{2}=r_{2} / K, b_{1}=r_{1} \alpha / K$, $b_{2}=r_{2} \beta / K$ in system (1)):

$$
\begin{aligned}
& \frac{\mathrm{d} x_{1}(t)}{\mathrm{d} t} \\
& =x_{1}(t)\left(r_{1}(t)-a_{1}(t) x_{1}(t)-b_{1}(t)\left[x_{2}(t)-c_{2}(t)\right]^{2}\right),
\end{aligned}
$$




$$
\begin{aligned}
& \frac{\mathrm{d} x_{2}(t)}{\mathrm{d} t} \\
& =x_{2}(t)\left(r_{2}(t)-a_{2}(t) x_{2}(t)-b_{1}(t)\left[x_{1}(t)-c_{1}(t)\right]^{2}\right) .
\end{aligned}
$$

In real world, the situation of enterprises is often distributed by unpredictable forces which can result in changes in enterprises' parameters such as intrinsic growth rates. So it is necessary to study models with control variables which are so-called disturbance functions [14-17].

As well known, both continuous and discrete systems are very important in implementation and applications, but it is troublesome to study the permanence and the existence of almost periodic solutions for continuous system and discrete system, respectively. It is very important to study that on time scales which can unify the continuous and discrete situations.

Motivated by the above statements, we consider the following competitive and cooperation model of a satellite enterprises and a dominant enterprise with feedback controls on time scale $\mathbb{T}$ :

$$
\begin{aligned}
x^{\Delta}(t)= & r_{1}(t)-a_{1}(t) \exp \{x(t)\} \\
& -b_{1}(t)\left[\exp \{y(t)\}-c_{2}(t)\right]^{2}-e_{1}(t) u(t), \\
u^{\Delta}(t)= & -\alpha_{1}(t) u(t)+\beta_{1}(t) \exp \{x(t)\}, \\
y^{\Delta}(t)= & r_{2}(t)-a_{2}(t) \exp \{y(t)\} \\
& +b_{2}(t)\left[\exp \{x(t)\}-c_{1}(t)\right]^{2}-e_{2}(t) v(t), \\
v^{\Delta}(t)= & -\alpha_{2}(t) v(t)+\beta_{2}(t) \exp \{y(t)\} .
\end{aligned}
$$

As pointed in $[18,19]$, periodic phenomenon and almost periodic phenomenon are widespread in nature and almost periodic phenomena is more frequent than periodic phenomenon. Hence, they have been the object of intensive analysis by numerous authors. In particular, there have been extensive results on existence of almost periodic solutions of differential equations in the literature. Some of these results can be found in [20-24]. Our main purpose of this paper is by using the comparison theorem of dynamic equations on time scales and constructing a suitable Lyapunov functional to study the permanence and the existence of almost periodic solutions of (3).

Remark 1. Let $N_{1}(t)=\exp \{x(t)\}, N_{2}(t)=\exp \{y(t)\}$; if $\mathbb{T}=\mathbb{R}$ (the set of all real numbers), then (3) reduces to

$$
\begin{aligned}
\frac{\mathrm{d} N_{1}(t)}{\mathrm{d} t}= & N_{1}(t)\left(r_{1}(t)-a_{1}(t) N_{1}(t)\right. \\
& -b_{1}(t)\left[N_{2}(t)-c_{2}(t)\right]^{2} \\
& \left.-e_{1}(t) u(t)\right),
\end{aligned}
$$

$$
\begin{aligned}
\frac{\mathrm{d} N_{2}(t)}{\mathrm{d} t}= & N_{2}(t)\left(r_{2}(t)-a_{2}(t) N_{2}(t)\right. \\
& -b_{1}(t)\left[N_{1}(t)-c_{1}(t)\right]^{2} \\
& \left.-e_{2}(t) v(t)\right), \\
\frac{\mathrm{d} u(t)}{\mathrm{d} t}=- & \alpha_{1}(t) u(t)+\beta_{1}(t) N_{1}(t), \\
\frac{\mathrm{d} v(t)}{\mathrm{d} t}=- & \alpha_{2}(t) v(t)+\beta_{2}(t) N_{2}(t) .
\end{aligned}
$$

If $\mathbb{T}=\mathbb{Z}$ (the set of all integers), then (3) reduces to

$$
\begin{gathered}
\begin{array}{c}
N_{1}(t+1)=N_{1}(t) \exp \left(r_{1}(t)-a_{1}(t) N_{1}(t)\right. \\
-b_{1}(t)\left[N_{2}(t)-c_{2}(t)\right]^{2} \\
\left.-e_{1}(t) u(t)\right), \\
N_{2}(t+1) \quad \\
=N_{2}(t) \exp \left(r_{2}(t)-a_{2}(t) N_{2}(t)+b_{2}(t)\left[N_{1}(t)-c_{1}(t)\right]^{2}\right. \\
\left.-e_{2}(t) v(t)\right), \\
\Delta u(t)=-\alpha_{1}(t) u(t)+\beta_{1}(t) N_{1}(t), \\
\Delta v(t)=-\alpha_{2}(t) v(t)+\beta_{2}(t) N_{2}(t) .
\end{array}
\end{gathered}
$$

Therefore, we only consider the permanence and existence of nonnegative almost periodic solution of system (3), then the permanence and almost periodic solution of (4) and (5) can be obtained as direct results of (3).

Let $\mathbb{T}$ and $\mathbb{T}^{+}$denote the nonempty closed subset (time scales) of $\mathbb{R}$. Let $f$ be a continuous bounded function defined on $\mathbb{T}$, and we denote

$$
f^{M}=\sup _{t \in \mathbb{T}}\{f(t)\}, \quad f^{m}=\inf _{t \in \mathbb{T}}\{f(t)\} .
$$

Throughout this paper, we assume that

$\left(\mathrm{H}_{1}\right) r_{i}(t), a_{i}(t), b_{i}(t), c_{i}(t), e_{i}(t), \alpha_{i}(t)$, and $\beta_{i}(t)$ are almost periodic functions on time scale $\mathbb{T}$ such that

$$
\begin{gathered}
0<r_{i}^{m} \leq r_{i}(t) \leq r_{i}^{M}, \quad 0<a_{i}^{m} \leq a_{i}(t) \leq a_{i}^{M}, \\
0<b_{i}^{m} \leq b_{i}(t) \leq b_{i}^{M},
\end{gathered}
$$




$$
\begin{gathered}
0<c_{i}^{m} \leq c_{i}(t) \leq c_{i}^{M}, \quad 0<e_{i}^{m} \leq e_{i}(t) \leq e_{i}^{M}, \\
0<\alpha_{i}^{m} \leq \alpha_{i}(t) \leq \alpha_{i}^{M}, \\
0<\beta_{i}^{m} \leq \beta_{i}(t) \leq \beta_{i}^{M}, \\
-a_{i}^{m} \in \mathscr{R}^{+}, \quad-\alpha_{i}^{m} \in \mathscr{R}^{+}, \quad i=1,2,
\end{gathered}
$$

where $\mathscr{R}^{+}$is the set of positively regressive functions from $\mathbb{T}$ to $\mathbb{R}$.

\section{Preliminaries}

In this section, we will recall some definitions and lemmas which will be used in the proof of our main results.

Let $\mathbb{T}$ be a nonempty closed subset (time scale) of $\mathbb{R}$. The forward and backward jump operators $\sigma, \rho: \mathbb{T} \rightarrow \mathbb{\mathbb { T }}$ and the graininess $\mu: \mathbb{T} \rightarrow \mathbb{R}^{+}$(the set of all nonnegative real numbers) are defined, respectively, by

$$
\begin{gathered}
\sigma(t)=\inf \{s \in \mathbb{T}: s>t\}, \\
\rho(t)=\sup \{s \in \mathbb{T}: s<t\}, \\
\mu(t)=\sigma(t)-t .
\end{gathered}
$$

A point $t \in \mathbb{T}$ is called left-dense if $t>\inf \mathbb{T}$ and $\rho(t)=t$, left-scattered if $\rho(t)<t$, right-dense if $t<\sup \mathbb{T}$ and $\sigma(t)=t$, and right-scattered if $\sigma(t)>t$. If $\mathbb{T}$ has a leftscattered maximum $m$, then $\mathbb{T}^{k}=\mathbb{T} \backslash\{m\}$; otherwise, $\mathbb{T}^{k}=\mathbb{T}$. If $\mathbb{T}$ has a right-scattered minimum $m$, then $\mathbb{T}_{k}=\mathbb{T} \backslash\{m\}$; otherwise $\mathbb{T}_{k}=\mathbb{T}$.

A function $f: \mathbb{T} \rightarrow \mathbb{R}$ is called $r d$-continuous provided it is continuous at right-dense points in $\mathbb{T}$ (i.e., $\lim _{s \in \mathbb{T}, s \rightarrow t} f(s)=$ $f(t))$ and its left-sided limits exist at left-dense points in $\mathbb{T}$. If $f$ is continuous at each right-dense point and each left-dense point, then $f$ is said to be a continuous function on $\mathbb{T}$. The set of continuous functions $f: \mathbb{T} \rightarrow \mathbb{R}$ will be denoted by $C(\mathbb{T})$.

For $y: \mathbb{T} \rightarrow \mathbb{R}$ and $t \in \mathbb{T}^{k}$; we define the delta derivative of $y(t), y^{\Delta}(t)$ to be the number (if it exists) with the property that, for any $\epsilon>0$, there exists a neighborhood $U$ of $t$ such that

$$
\left|[y(\sigma(t))-y(s)]-y^{\Delta}(t)[\sigma(t)-s]\right|<\epsilon|\sigma(t)-s|
$$

for all $s \in U$. Let $y$ be right-dense continuous. If $Y^{\Delta}(t)=y(t)$, then we define the delta integral by

$$
\int_{a}^{t} y(s) \Delta(s)=Y(t)-Y(a)
$$

A function $p: \mathbb{T} \rightarrow \mathbb{R}$ is called regressive provided $1+\mu(t) p(t) \neq 0$ for all $t \in \mathbb{T}^{k}$. The set of all regressive and rd-continuous functions $p: \mathbb{T} \rightarrow \mathbb{R}$ will be denoted by $\mathscr{R}=\mathscr{R}(\mathbb{T})=\mathscr{R}(\mathbb{T}, R)$. If $1+\mu(t) p(t)>0, \forall t \in \mathbb{T}$, then $p$ is a positively regressive function from $\mathbb{T}$ to $\mathbb{R}$. If $r$ is a regressive function, then the generalized exponential function $e_{r}$ is defined by

$$
e_{r}(t, s)=\exp \left\{\int_{s}^{t} \xi_{\mu(\tau)}(r(\tau)) \Delta \tau\right\}, \quad s, t \in \mathbb{T}
$$

with the cylinder transformation

$$
\xi_{h}(z)= \begin{cases}\frac{\log (1+h z)}{h}, & \text { if } h \neq 0 \\ z, & \text { if } h=0\end{cases}
$$

Definition 2 (see $[25,26])$. Let $p, q: \mathbb{T} \rightarrow \mathscr{R}$ be two regressive functions, we define

$$
\begin{gathered}
p \oplus q=p+q+\mu p q, \quad \ominus p=-\frac{p}{1+\mu p}, \\
p \ominus q=p \oplus(\ominus q) .
\end{gathered}
$$

Then the generalized exponential function has the following properties.

Lemma 3 (see $[25,26]$ ). Assume that $p, q: \mathbb{T} \rightarrow \mathbb{R}$ are two regressive functions; then

(i) $e_{0}(t, s) \equiv 1$ and $e_{p}(t, t) \equiv 1$;

(ii) $e_{p}(\sigma(t), s)=(1+\mu(t) p(t)) e_{p}(t, s)$;

(iii) $e_{p}(t, s)=1 / e_{p}(s, t)=e_{\ominus p}(s, t)$;

(iv) $e_{p}(t, s) e_{p}(s, r)=e_{p}(t, r)$;

(v) $e_{p}(t, s) e_{p}(s, r)=e_{p}(t, r)$;

(vi) If $a, b, c \in \mathbb{T}$, then $\int_{a}^{b} e_{p}(c, \sigma(t)) \Delta(t)=e_{p}(c, a)-$ $e_{p}(c, b)$.

Definition 4 (see [27]). A time scale $\mathbb{T}$ is called an almost periodic time scale if

$$
\Pi:=\{\tau \in \mathbb{R}: t+\tau \in \mathbb{T}, \forall t \in \mathbb{T}\} \neq\{0\} .
$$

Throughout this paper, we restrict our discussion on almost periodic time scales.

Definition 5 (see [27]). A function $f: \mathbb{T} \rightarrow \mathbb{R}$ is called an almost periodic function if the $\epsilon$-translation number set of $f$ :

$$
T(f, \epsilon, \mathbb{T})=\{\tau \in \mathbb{T}:|f(t+\tau)-f(t)|<\epsilon, \forall t \in \mathbb{T}\}
$$

is a relatively dense set in $\mathbb{T}$ for all $\epsilon>0$; that is, for any given $\epsilon>0$, there exists a number $l=l(\epsilon)>0$ such that for any interval with length $l(\epsilon)$, there is a number $\tau=\tau(\epsilon) \epsilon$ $T(f, \epsilon, \mathbb{T})>0$ in this interval such that

$$
|f(t+\tau)-f(t)|<\epsilon, \quad t \in \mathbb{T} .
$$

Definition 6 (see [27]). Let $f: \mathbb{T} \times \mathbb{D} \rightarrow \mathbb{R}$, where $\mathbb{D}$ is an open set in $\mathbb{R}$ and $f(t, x)$ is said to be almost periodic in $t$ uniformly for $x \in \mathbb{D}$ or uniformly almost periodic for short. If for any compact set $\mathbb{S}$ in $\mathbb{D}$, there exists a positive number $l(\epsilon, s)$ such that any interval of length $l(\epsilon, s)$ contains a number $\tau$ for which $|f(t+\tau, x)-f(t, x)|<\epsilon$ for all $t \in \mathbb{T}$ and $x \in \mathbb{S}$, $\tau$ is called the $\epsilon$-translation number of $f(t, x)$. 
Lemma 7 (see [27]). A $f(t)$ is an almost periodic function if and only iffor any sequence $\left\{\tau_{n}^{\prime}\right\} \subset \mathbb{T}$ there exists a subsequence $\left\{\tau_{n}\right\} \subset\left\{\tau_{n}^{\prime}\right\}$ such that $f\left(t+\tau_{n}\right)$ converges uniformly on $t \in \mathbb{T}$ as $n \rightarrow \infty$. Furthermore, the limit function is also an almost periodic function.

Definition 8. System (3) is said to be permanent if there exist positive constants $M_{i}, N_{i}, m_{i}, n_{i}(i=1,2,3,4)$ which are independent of the solutions of the system, such that any solution $(x(t), y(t), u(t), v(t))^{T}$ of system (3) satisfies

$$
\begin{aligned}
& m_{1} \leq \liminf _{t \rightarrow \infty} x(t) \leq \limsup _{t \rightarrow \infty} x(t) \leq M_{1}, \\
& m_{2} \leq \liminf _{t \rightarrow \infty} y(t) \leq \limsup _{t \rightarrow \infty} y(t) \leq M_{2}, \\
& m_{3} \leq \liminf _{t \rightarrow \infty} u(t) \leq \limsup _{t \rightarrow \infty} u(t) \leq M_{3}, \\
& m_{4} \leq \liminf _{t \rightarrow \infty} v(t) \leq \limsup _{t \rightarrow \infty} v(t) \leq M_{4} .
\end{aligned}
$$

Consider the following nonlinear almost periodic differential system on time scales:

$$
x^{\Delta}=f(t, x)
$$

and the product system of (18)

$$
x^{\Delta}=f(t, x), \quad y^{\Delta}=f(t, y) .
$$

Lemma 9 (see $[28,29])$. Suppose that there exists a Lyapunov functional $V(t, x, y)$ defined for $V(t, x, y) \in\left(\mathbb{T}^{+} \times \mathbb{D} \times \mathbb{D}, \mathbb{R}\right)$ satisfying the following conditions:

(i) $a(|x-y|) \leq V(t, x, y) \leq b(|x-y|)$, where $a, b \in k, k=$ $\left\{a \in c\left(\mathscr{R}^{+}, \mathscr{R}^{+}\right): a(0)=0\right.$ and $a$ is increasing $\}$

(ii) $\left|V\left(t, x_{1}, y_{1}\right)-V\left(t, x_{2}, y_{2}\right)\right| \leq L\left[\left|x_{1}-x_{2}\right|+\left|y_{1}-y_{2}\right|\right]$, where $L>0$ is a constant;

(iii) $D^{+} V_{(19)}^{\Delta}(t, x, y) \leq-c V(t, x, y)$, where $-c \in \mathscr{R}^{+}$and $c>0$.

In addition, if there exists a solution $x(t)$ of system (18) such that $x(t) \in \mathbb{S}$ for $t \in \mathbb{T}^{+}$, where $\mathbb{S} \subset \mathbb{D}$ is any compact subset of $\mathbb{D}$, then there exists a unique uniformly asymptotically stable almost periodic solution $p(t)$ of system (18). In particular, if $f(t, x)$ is periodic of period $\omega$, then there exists a unique uniformly asymptotically stable periodic solution of (18) of period $\omega$.

\section{Persistence}

We need the following lemma to obtain the permanence of system (3).

Lemma 10 (see $[28,29])$. Let $-a \in \mathscr{R}^{+}$.

(i) If $x^{\Delta}(t) \leq b-a x(t)$, then for $t>t_{0}$

$$
\begin{aligned}
x(t) \leq & x\left(t_{0}\right) e_{(-a)}\left(t, t_{0}\right) \\
& +\frac{b}{a}\left(1-e_{(-a)}\left(t, t_{0}\right)\right) .
\end{aligned}
$$

In particular, if $a>0, b>0$, we have $\lim \sup _{t \rightarrow+\infty} x(t) \leq b / a$.

(ii) If $x^{\Delta}(t) \geq b-a x(t)$, then for $t>t_{0}$

$$
\begin{aligned}
x(t) \geq & x\left(t_{0}\right) e_{(-a)}\left(t, t_{0}\right) \\
& +\frac{b}{a}\left(1-e_{(-a)}\left(t, t_{0}\right)\right) .
\end{aligned}
$$

In particular, if $a>0, b>0$, we have $\lim \inf _{t \rightarrow+\infty} x(t) \geq b / a$.

Proposition 11. Assume that $\left(H_{1}\right)$ holds; then every solution $(x(t), y(t), u(t), v(t))^{T}$ of system (3) satisfies

$$
\begin{array}{ll}
\limsup _{t \rightarrow \infty} x(t) \leq x^{*}, & \limsup _{t \rightarrow \infty} y(t) \leq y^{*}, \\
\limsup _{t \rightarrow \infty} u(t) \leq u^{*}, & \limsup _{t \rightarrow \infty} v(t) \leq v^{*},
\end{array}
$$

where

$$
\begin{gathered}
x^{*}=\frac{r_{1}^{M}-a_{1}^{m}}{a_{1}^{m}}, \quad y^{*}=\frac{r_{2}^{M}-a_{2}^{m}+b_{2}^{M} e^{2 x^{*}}}{a_{2}^{m}}, \\
u^{*}=\frac{\beta_{1}^{M} e^{x^{*}}}{\alpha_{1}^{m}}, \quad v^{*}=\frac{\beta_{2}^{M} e^{y^{*}}}{\alpha_{2}^{m}} .
\end{gathered}
$$

Proof. Let $(x(t), y(t), u(t), v(t))^{T}$ be any solution of system (3); it follows from the first equation of system (3) and the Bernoulli inequality $e^{x} \geq 1+x$ for $x \in \mathbb{R}$, that

$$
\begin{aligned}
x^{\Delta}(t) & \leq r_{1}(t)-a_{1}(t) \exp x(t) \\
& \leq r_{1}(t)-a_{1}(t)(1+x(t)) \\
& \leq\left(r_{1}^{M}-a_{1}^{m}\right)-a_{1}^{m} x(t) .
\end{aligned}
$$

By applying (i) of Lemma 10 to the differential inequality above, we have

$$
\limsup _{t \rightarrow \infty} x(t) \leq \frac{r_{1}^{M}-a_{1}^{m}}{a_{1}^{m}}:=x^{*} .
$$

For any positive constant $\epsilon$ small enough, it follows from (25) that there exists a large enough $T_{0}>0$ such that $x(t) \leq$ $x^{*}+\epsilon$ for all $t>T_{0}$. Then the second equation and the third equation of system (3) lead to

$$
\begin{aligned}
u^{\Delta}(t) & \leq-\alpha_{1}^{m} u(t)+\beta_{1}^{M} e^{x^{*}+\epsilon} \\
y^{\Delta}(t) & \leq r_{2}(t)-a_{2}(t) \exp y(t)+b_{2}(t) e^{2\left(x^{*}+\epsilon\right)} \\
& \leq r_{2}(t)-a_{2}(t)(y(t)+1)+b_{2}(t) e^{2\left(x^{*}+\epsilon\right)} .
\end{aligned}
$$

That is,

$$
\begin{aligned}
& u^{\Delta}(t) \leq-\alpha_{1}^{m} u(t)+\beta_{1}^{M} e^{x^{*}+\epsilon} \\
& y^{\Delta}(t) \leq r_{2}^{M}+b_{2}^{M} e^{2\left(x^{*}+\epsilon\right)}-a_{2}^{m}-a_{2}^{m} y(t) .
\end{aligned}
$$


By applying Lemma 10, it follows from (27) that

$$
\begin{aligned}
& \limsup _{t \rightarrow \infty} y(t) \leq \frac{r_{2}^{M}-a_{2}^{m}+b_{2}^{M} e^{2 x^{*}+\epsilon}}{a_{2}^{m}}, \\
& \limsup _{t \rightarrow \infty} u(t) \leq \frac{\beta_{1}^{M} e^{x^{*}+\epsilon}}{\alpha_{1}^{m}} .
\end{aligned}
$$

Letting $\epsilon \rightarrow 0$ in the above inequality leads to

$$
\begin{gathered}
\limsup _{t \rightarrow \infty} y(t) \leq \frac{r_{2}^{M}-a_{2}^{m}+b_{2}^{M} e^{2 x^{*}}}{a_{2}^{m}}:=y^{*}, \\
\limsup _{t \rightarrow \infty} u(t) \leq \frac{\beta_{1}^{M} e^{x^{*}}}{\alpha_{1}^{m}}:=u^{*} .
\end{gathered}
$$

By using similar arguments as those in the proofs of (28), it follows that

$$
\limsup _{t \rightarrow \infty} v(t) \leq \frac{\beta_{2}^{M} e^{y^{*}}}{\alpha_{2}^{m}}:=v^{*}
$$

This completes the proof.

Proposition 12. Assume that $\left(H_{1}\right)$ holds; suppose further that

$\left(\mathrm{H}_{2}\right) r_{1}^{m}-b_{1}^{M}\left(e^{y^{*}}-c_{2}^{m}\right)^{2}-e_{1}^{M} u^{*}>0, r_{2}^{m}-e_{2}^{M} v^{*}>$ 0 , then every solution $(x(t), y(t), u(t), v(t))^{T}$ of system (3) satisfies

$$
\begin{array}{ll}
\liminf _{t \rightarrow \infty} x(t) \geq x_{*}, & \liminf _{t \rightarrow \infty} y(t) \geq y_{*}, \\
\liminf _{t \rightarrow \infty} u(t) \geq u_{*}, & \liminf _{t \rightarrow \infty} v(t) \geq v_{*},
\end{array}
$$

where

$$
\begin{gathered}
x_{*}=\ln \left(\frac{r_{1}^{m}-b_{1}^{M}\left[e^{y^{*}}-c_{2}^{m}\right]^{2}-e_{1}^{M} u^{*}}{a_{1}^{M}}\right), \\
y_{*}=\ln \left(\frac{r_{2}^{m}-e_{2}^{M} v^{*}+b_{2}^{m}\left[e^{x_{*}}-c_{1}^{M}\right]^{2}}{a_{2}^{M}}\right), \\
u_{*}=\frac{\beta_{1}^{m} e^{x_{*}}}{\alpha_{1}^{M}}, \quad v_{*}=\frac{\beta_{2}^{m} e^{y_{*}}}{\alpha_{2}^{M}} .
\end{gathered}
$$

Proof. Let $(x(t), y(t), u(t), v(t))^{T}$ be any positive solution of system (3). We first prove that

$$
\liminf _{t \rightarrow \infty} x(t) \geq x_{*}
$$

For any small enough constant $\epsilon \geq 0$, there exists a point $T_{1} \epsilon$
$\mathbb{T}$ and $T_{1} \geq T_{0}$ such that

$$
\begin{gathered}
x(t) \leq x^{*}+\epsilon, \quad y(t) \leq y^{*}+\epsilon \\
u(t) \leq u^{*}+\epsilon, \quad v(t) \leq v^{*}+\epsilon, \quad t>T_{1} .
\end{gathered}
$$

By the first equation of system (3) and condition $\left(\mathrm{H}_{1}\right)$, we have

$$
\begin{aligned}
x^{\Delta}(t) \geq & r_{1}^{m}-a_{1}^{M} \exp \{x(t)\}-b_{1}^{M}\left[\exp \left\{y^{*}+\epsilon\right\}-c_{2}^{m}\right]^{2} \\
& -e_{1}^{M}\left(u^{*}+\epsilon\right) \\
\geq & r_{1}^{m}-b_{1}^{M}\left[e^{y^{*}+\epsilon}-c_{2}^{m}\right]^{2}-e_{1}^{M}\left(u^{*}+\epsilon\right) \\
& -a_{1}^{M} \exp \{x(t)\}, \quad t>T_{1} .
\end{aligned}
$$

We assert

$$
\begin{aligned}
& r_{1}^{m}-b_{1}^{M}\left[e^{y^{*}+\epsilon}-c_{2}^{m}\right]^{2}-e_{1}^{M}\left(u^{*}+\epsilon\right)-a_{1}^{M} \exp \{x(t)\} \\
& \leq 0, \quad t>T_{1}
\end{aligned}
$$

otherwise, we assume that there exists $\tilde{t}>T_{1}$ such that

$$
\begin{aligned}
& r_{1}^{m}-b_{1}^{M}\left[e^{y^{*}+\epsilon}-c_{2}^{m}\right]^{2}-e_{1}^{M}\left(u^{*}+\epsilon\right)-a_{1}^{M} \exp \{x(\widetilde{t})\} \\
& >0 \\
& r_{1}^{m}-b_{1}^{M}\left[e^{y^{*}+\epsilon}-c_{2}^{m}\right]^{2}-e_{1}^{M}\left(u^{*}+\epsilon\right)-a_{1}^{M} \exp \{x(t)\} \\
& \leq 0, \quad \forall t \in\left[T_{1}, \widetilde{t}\right),
\end{aligned}
$$

then we get

$x(\widetilde{t})$

$$
<\ln \left(\frac{r_{1}^{m}-b_{1}^{M}\left[e^{y^{*}+\epsilon}-c_{2}^{m}\right]^{2}-e_{1}^{M}\left(u^{*}+\epsilon\right)}{a_{1}^{M}}\right),
$$

$x(t)$

$$
\geq \ln \left(\frac{r_{1}^{m}-b_{1}^{M}\left[e^{y^{*}+\epsilon}-c_{2}^{m}\right]^{2}-e_{1}^{M}\left(u^{*}+\epsilon\right)}{a_{1}^{M}}\right),
$$

$\forall t \in\left[T_{1}, \tilde{t}\right)$. 
Equation (38) implies $x^{\Delta}(\tilde{t}) \leq 0$, which is a contradiction; therefore (36) holds for $\forall t \geq T_{1}$.

Consequently,

$$
x(t) \geq \ln \left(\frac{r_{1}^{m}-b_{1}^{M}\left[e^{y^{*}+\epsilon}-c_{2}^{m}\right]^{2}-e_{1}^{M}\left(u^{*}+\epsilon\right)}{a_{1}^{M}}\right),
$$

$$
\forall t \geq T_{1}
$$

then

$$
\lim _{t \rightarrow+\infty} \inf _{t}(t) \geq \ln \left(\frac{r_{1}^{m}-b_{1}^{M}\left[e^{y^{*}}-c_{2}^{m}\right]^{2}-e_{1}^{M} u^{*}}{a_{1}^{M}}\right)=x_{*} .
$$

Now, for any small enough $\epsilon>0$, there exists a point $T_{2} \in \mathbb{T}$ such that $x(t) \geq x_{*}-\epsilon, t \geq T_{2}$; from the third equation of system (3), we have

$$
\begin{aligned}
y^{\Delta}(t) \geq & r_{2}^{m}-a_{2}^{M} \exp \{y(t)\}+b_{2}^{m}\left[\exp \left\{x_{*}-\epsilon\right\}-c_{1}^{M}\right]^{2} \\
& -e_{2}^{M}\left(v^{*}+\epsilon\right)
\end{aligned}
$$

by using a similar argument as that in (40), we can get

$$
\liminf _{t \rightarrow+\infty} y(t) \geq \ln \left(\frac{r_{2}^{m}+b_{2}^{m}\left[e^{x_{*}}-c_{1}^{M}\right]^{2}-e_{2}^{M} v^{*}}{a_{2}^{M}}\right):=y_{*} .
$$

Next we prove

$$
\liminf _{t \rightarrow \infty} u(t) \geq u_{*}, \quad \liminf _{t \rightarrow \infty} v(t) \geq v_{*} .
$$

Since $\lim \inf _{t \rightarrow \infty} x(t) \geq x_{*}$ then there exists $T \in \mathbb{T}$ such that $x(t) \geq x_{*}-\epsilon$ for any $\epsilon>0$ and $t>T$. By the second equation of system (3), we have

$$
u^{\Delta}(t) \geq-\alpha_{1}^{M} u(t)+\beta_{1}^{m} e^{x_{*}-\varepsilon},
$$

Applying (ii) of Lemma 10, we have

$$
\liminf _{t \rightarrow \infty} u(t) \geq \frac{\beta_{1}^{m} e^{x_{*}}}{\alpha_{1}^{M}}:=u_{*} ;
$$

similar to the above proof, it is easy to obtain that

$$
\liminf _{t \rightarrow \infty} v(t) \geq \frac{\beta_{2}^{m} e^{y_{*}}}{\alpha_{2}^{M}}:=v_{*} .
$$

So the proof of Proposition 12 is complete.

Theorem 13. Assume that $\left(H_{1}\right)-\left(H_{2}\right)$ hold; then system (3) is persistent.

We denote by $\Omega$ the set of all solutions $(x(t), y(t), u(t), v(t))^{T}$ of system (3) satisfying

$$
\begin{array}{ll}
x_{*} \leq x(t) \leq x^{*}, & y_{*} \leq y(t) \leq y^{*}, \\
u_{*} \leq u(t) \leq u^{*}, & v_{*} \leq v(t) \leq v^{*},
\end{array}
$$

for all $t \in \mathbb{T}$. It should be noticed that, from the proofs of Propositions 11 and 12, we know that the conditions of Theorem 13 hold and $\Omega$ is an invariant set of system (3).

\section{Existence of Uniformly Asymptotically Stable Almost Periodic Solution}

According to Lemma 9, we first prove that there exists a bounded solution of system (3) and then construct an adaptive Lyapunov functional for system (3). The next result tells us that there exists a bounded solution of (3). To be precise, consider the following.

Proposition 14. Assume that $\left(H_{1}\right)-\left(H_{2}\right)$ hold, then $\Omega \neq \emptyset$.

Proof. By the almost periodicity of $r_{i}(t), a_{i}(t), b_{i}(t), c_{i}(t), e_{i}(t)$, $\alpha_{i}(t), \beta_{i}(t), i=1,2$, there exists a sequence $\left\{\tau_{n}\right\} \subseteq \mathbb{V}$ with $\tau_{n} \rightarrow \infty$ as $n \rightarrow \infty$ such that

$$
\begin{gathered}
r_{i}\left(t+\tau_{n}\right) \longrightarrow r_{i}(t), \quad a_{i}\left(t+\tau_{n}\right) \longrightarrow a_{i}(t), \\
b_{i}\left(t+\tau_{n}\right) \longrightarrow b_{i}(t), \quad c_{i}\left(t+\tau_{n}\right) \longrightarrow c_{i}(t), \\
e_{i}\left(t+\tau_{n}\right) \longrightarrow e_{i}(t), \quad \alpha_{i}\left(t+\tau_{n}\right) \longrightarrow \alpha_{i}(t), \\
\beta_{i}\left(t+\tau_{n}\right) \longrightarrow \beta_{i}(t), \quad n \longrightarrow \infty .
\end{gathered}
$$

Let $\epsilon$ be an arbitrary small number; it follows from Propositions 11 and 12 that there exists a positive number $T_{3}$ such that

$$
\begin{gathered}
x_{*}-\epsilon \leq x(t) \leq x^{*}+\epsilon, \\
y_{*}-\epsilon \leq y(t) \leq y^{*}+\epsilon, \\
u_{*}-\epsilon \leq u(t) \leq u^{*}+\epsilon, \\
v_{*}-\epsilon \leq v(t) \leq v^{*}+\epsilon \quad \forall t>T_{3} .
\end{gathered}
$$

Denote

$$
\begin{aligned}
x_{n}(t):=x\left(t+\tau_{n}\right), & y_{n}(t):=y\left(t+\tau_{n}\right), \\
u_{n}(t):=u\left(t+\tau_{n}\right), & v_{n}(t):=v\left(t+\tau_{n}\right), \\
t \geq T_{3}-\tau_{n}, & n=1,2, \ldots
\end{aligned}
$$

For any positive integer $m$, it is easy to see that there exist sequences

$$
\begin{array}{ll}
\left\{x_{n}(t): n \geq m\right\}, & \left\{y_{n}(t): n \geq m\right\}, \\
\left\{u_{n}(t): n \geq m\right\}, & \left\{v_{n}(t): n \geq m\right\}
\end{array}
$$

such that the sequences $\left\{x_{n}(t)\right\},\left\{y_{n}(t)\right\},\left\{u_{n}(t)\right\},\left\{v_{n}(t)\right\}$ have subsequences, denoted by $\left\{x_{n}(t)\right\},\left\{y_{n}(t)\right\},\left\{u_{n}(t)\right\},\left\{v_{n}(t)\right\}$ again, converging on any finite interval of $\mathbb{T}$ as $n \rightarrow \infty$, respectively. Thus we have functions $x^{*}(t), y^{*}(t), u^{*}(t), v^{*}(t)$ such that

$$
\begin{gathered}
x_{n}(t) \longrightarrow x^{*}(t), \quad y_{n}(t) \longrightarrow y^{*}(t), \\
u_{n}(t) \longrightarrow u^{*}(t), \quad v_{n}(t) \longrightarrow v^{*}(t), \quad t \in \mathbb{T}, n \longrightarrow \infty .
\end{gathered}
$$


Combining with

$$
\begin{aligned}
x^{\Delta}(t)= & r_{1}\left(t+\tau_{n}\right)-a_{1}\left(t+\tau_{n}\right) \exp \left\{x\left(t+\tau_{n}\right)\right\} \\
& -b_{1}\left(t+\tau_{n}\right)\left[\exp \left\{y\left(t+\tau_{n}\right)\right\}-c_{2}\left(t+\tau_{n}\right)\right]^{2} \\
& -e_{1}\left(t+\tau_{n}\right) u\left(t+\tau_{n}\right), \\
u^{\Delta}(t)= & -\alpha_{1}\left(t+\tau_{n}\right) u\left(t+\tau_{n}\right)+\beta_{1}\left(t+\tau_{n}\right) \exp \left\{x\left(t+\tau_{n}\right)\right\}, \\
y^{\Delta}(t)= & r_{2}\left(t+\tau_{n}\right)-a_{2}\left(t+\tau_{n}\right) \exp \left\{y\left(t+\tau_{n}\right)\right\} \\
& +b_{2}\left(t+\tau_{n}\right)\left[\exp \left\{x\left(t+\tau_{n}\right)\right\}-c_{1}\left(t+\tau_{n}\right)\right]^{2} \\
& -e_{2}\left(t+\tau_{n}\right) v\left(t+\tau_{n}\right), \\
v^{\Delta}(t)= & -\alpha_{2}\left(t+\tau_{n}\right) v\left(t+\tau_{n}\right)+\beta_{2}\left(t+\tau_{n}\right) \exp \left\{y\left(t+\tau_{n}\right)\right\}
\end{aligned}
$$

gives

$$
\begin{aligned}
x^{* \Delta}(t)= & r_{1}(t)-a_{1}(t) \exp \left\{x^{*}(t)\right\} \\
& -b_{1}(t)\left[\exp \left\{y^{*}(t)\right\}-c_{2}(t)\right]^{2}-e_{1}(t) u^{*}(t), \\
u^{* \Delta}(t)= & -\alpha_{1}(t) u^{*}(t)+\beta_{1}(t) \exp \left\{x^{*}(t)\right\}, \\
y^{* \Delta}(t)= & r_{2}(t)-a_{2}(t) \exp \left\{y^{*}(t)\right\} \\
& +b_{2}(t)\left[\exp \left\{x^{*}(t)\right\}-c_{1}(t)\right]^{2}-e_{2}(t) v^{*}(t), \\
v^{* \Delta}(t)= & -\alpha_{2}(t) v^{*}(t)+\beta_{2}(t) \exp \left\{y^{*}(t)\right\} .
\end{aligned}
$$

We can easily see that $\left(x^{*}(t), y^{*}(t), u^{*}(t), v^{*}(t)\right)^{T}$ is a solution of system (3) and

$$
\begin{aligned}
& x_{*}-\epsilon \leq x^{*}(t) \leq x^{*}+\epsilon, \\
& y_{*}-\epsilon \leq y^{*}(t) \leq y^{*}+\epsilon, \\
& u_{*}-\epsilon \leq u^{*}(t) \leq u^{*}+\epsilon, \\
& v_{*}-\epsilon \leq v^{*}(t) \leq v^{*}+\epsilon,
\end{aligned}
$$

for $t \in \mathbb{T}$. Since $\epsilon$ is an arbitrary small positive number, it follows that

$$
\begin{array}{ll}
x_{*} \leq x^{*}(t) \leq x^{*}, & y_{*} \leq y^{*}(t) \leq y^{*}, \\
u_{*} \leq u^{*}(t) \leq u^{*}, & v_{*} \leq v^{*}(t) \leq v^{*}
\end{array}
$$

for $t \in \mathbb{T}$. This completes the proof.

Theorem 15. Assume that $\left(H_{1}\right)-\left(H_{2}\right)$ hold. Suppose further that
$\left(\mathrm{H}_{3}\right) c>0$ and $-c \in \mathscr{R}^{+}$, where $c=\min \left\{P_{1}-Q_{1} \mu, P_{2}-\right.$ $\left.Q_{2} \mu, R_{1}-S_{1} \mu, R_{2}-S_{2} \mu\right\}$,

$$
\begin{aligned}
& P_{1}=2 a_{1}^{m} \xi_{1}^{m}-b_{1}^{m} \xi_{2}^{m}\left(2 e^{y_{*}}-2 c_{2}^{M}\right)-e_{1}^{m} \\
& -b_{2}^{M} \xi_{2}^{M}\left(2 e^{x^{*}}-2 c_{1}^{m}\right)-\beta_{1}^{M} \xi_{1}^{M}, \\
& Q_{1}=a_{1}^{M^{2}} \xi_{1}^{M^{2}}+b_{1}^{M} a_{1}^{M} \xi_{1}^{M} \xi_{2}^{M}\left[2 e^{y^{*}}-2 c_{2}^{m}\right] \\
& +a_{1}^{M} \xi_{1}^{M} e_{1}^{M}+b_{2}^{M^{2}} \xi_{1}^{M^{2}}\left[2 e^{x^{*}}-2 c_{1}^{m}\right]^{2} \\
& +b_{2}^{M} e_{2}^{M} \xi_{1}^{M}\left[2 e^{x^{*}}-2 c_{1}^{m}\right]+\beta_{1}^{M^{2}} \xi_{2}^{M^{2}} \\
& +a_{2}^{m} b_{2}^{m} \xi_{1}^{m} \xi_{2}^{m}\left[2 e^{x^{*}}-2 c_{1}^{M}\right]+\beta_{1}^{m} \xi_{1}^{m} \alpha_{1}^{m}, \\
& P_{2}=2 a_{2}^{m} \xi_{2}^{m}-b_{1}^{m} \xi_{2}^{m}\left(2 e^{y_{*}}-2 c_{2}^{M}\right) \\
& -e_{2}^{m}-b_{2}^{M} \xi_{2}^{M}\left(2 e^{x^{*}}-2 c_{1}^{m}\right)-\beta_{2}^{M} \xi_{2}^{M}, \\
& Q_{2}=a_{2}^{M^{2}} \xi_{2}^{M^{2}}+b_{1}^{M} a_{1}^{M} \xi_{1}^{M} \xi_{2}^{M}\left[2 e^{y^{*}}-2 c_{2}^{m}\right] \\
& +b_{1}^{M^{2}} \xi_{2}^{M^{2}}\left[2 e^{y^{*}}-2 c_{2}^{m}\right]^{2}+b_{1}^{M} e_{1}^{M} \xi_{2}^{M}\left[2 e^{y^{*}}-2 c_{2}^{m}\right] \\
& +a_{2}^{m} \xi_{2}^{m} b_{2}^{m} \xi_{1}^{m}\left[2 e^{x_{*}}-2 c_{1}^{M}\right]+a_{2}^{M} \xi_{2}^{M} e_{2}^{M} \\
& +\beta_{2}^{M^{2}} \xi_{2}^{M^{2}}+\beta_{2}^{m} \xi_{2}^{m} \alpha_{2}^{m} \text {, } \\
& R_{i}=2 \alpha_{i}^{m}-\beta_{i}^{M} \xi_{i}^{M}-e_{i}^{m}, \quad(i=1,2), \\
& S_{1}=e_{1}^{M^{2}}+e_{1}^{M} b_{1}^{M} \xi_{2}^{M}\left(2 e^{y^{*}}-2 c_{2}^{m}\right) \\
& +a_{1}^{M} \xi_{1}^{M} e_{1}^{M}+\alpha_{1}^{M^{2}}+\beta_{1}^{m} \xi_{1}^{m} \alpha_{1}^{m}, \\
& S_{2}=e_{2}^{M^{2}}+e_{2}^{M} b_{2}^{M} \xi_{1}^{M}\left(2 e^{x^{*}}-2 c_{1}^{m}\right) \\
& +a_{2}^{M} \xi_{2}^{M} e_{2}^{M}+\alpha_{2}^{M^{2}}+\beta_{2}^{m} \xi_{2}^{m} \alpha_{2}^{m}, \\
& \mu=\sup _{t \in \mathbb{T}} \mu(t), \quad \xi_{1}^{m}=e^{x_{*}}, \quad \xi_{1}^{M}=e^{x^{*}}, \\
& \xi_{2}^{m}=e^{y_{*}}, \quad \xi_{2}^{M}=e^{y^{*}}
\end{aligned}
$$

then (3) has a unique almost periodic solution $X(t)=$ $(x(t), y(t), u(t), v(t))^{T}$ that is uniformly asymptotically stable and satisfies

$$
\begin{array}{cc}
x_{*} \leq x(t) \leq x^{*}, & y_{*} \leq y(t) \leq y^{*}, \\
u_{*} \leq u(t) \leq u^{*}, & v_{*} \leq v(t) \leq v^{*}
\end{array}
$$

for all $t \in \mathbb{T}$. 
Proof. From Proposition 14, we know that system (3) has a bounded solution satisfying

$$
\begin{gathered}
x_{*} \leq x(t) \leq x^{*}, \quad y_{*} \leq y(t) \leq y^{*}, \\
u_{*} \leq u(t) \leq u^{*}, \quad v_{*} \leq v(t) \leq v^{*}, \quad t \in \mathbb{T} .
\end{gathered}
$$

Hence,

$$
\begin{array}{ll}
|x(t)|<A_{1}, & |y(t)|<A_{2}, \\
|u(t)|<A_{3}, & |v(t)|<A_{4},
\end{array}
$$

where

$$
\begin{array}{ll}
A_{1}=\max \left\{x_{*}, x^{*}\right\}, & A_{2}=\max \left\{y_{*}, y^{*}\right\}, \\
A_{3}=\max \left\{u_{*}, u^{*}\right\}, & A_{4}=\max \left\{v_{*}, v^{*}\right\} .
\end{array}
$$

For all $(x, y, u, v) \in \mathbb{R}^{4}$, we define the norm

$$
\|(x, y, u, v)\|=|x|+|y|+|u|+|v| .
$$

Suppose that $X=(x(t), y(t), u(t), v(t))$ and $Y=$ $(p(t), q(t), w(t), z(t))$ are any two solutions of system (3) defined on $\mathbb{T}^{+} \times \Omega^{2}$; then $\|X\| \leq C,\|Y\| \leq C$, where $C=\sum_{1}^{4} A_{i}$.

Consider the product system of system (3)

$$
\begin{aligned}
x^{\Delta}(t)= & r_{1}(t)-a_{1}(t) \exp \{x(t)\} \\
& -b_{1}(t)\left[\exp \{y(t)\}-c_{2}(t)\right]^{2}-e_{1}(t) u(t), \\
u^{\Delta}(t)= & -\alpha_{1}(t) u(t)+\beta_{1}(t) \exp \{x(t)\}, \\
y^{\Delta}(t)= & r_{2}(t)-a_{2}(t) \exp \{y(t)\} \\
& +b_{2}(t)\left[\exp \{x(t)\}-c_{1}(t)\right]^{2}-e_{2}(t) v(t), \\
v^{\Delta}(t)= & -\alpha_{2}(t) v(t)+\beta_{2}(t) \exp \{y(t)\} \\
p^{\Delta}(t)= & r_{1}(t)-a_{1}(t) \exp \{p(t)\} \\
& -b_{1}(t)\left[\exp \{q(t)\}-c_{2}(t)\right]^{2}-e_{1}(t) w(t), \\
w^{\Delta}(t)= & -\alpha_{1}(t) w(t)+\beta_{1}(t) \exp \{p(t)\} \\
q^{\Delta}(t)= & r_{2}(t)-a_{2}(t) \exp \{q(t)\} \\
& +b_{2}(t)\left[\exp \{p(t)\}-c_{1}(t)\right]^{2}-e_{2}(t) z(t), \\
z^{\Delta}(t)= & -\alpha_{2}(t) z(t)+\beta_{2}(t) \exp \{q(t)\}
\end{aligned}
$$

We construct a Lyapunov function defined on $\mathbb{T}^{+} \times \Omega^{2}$ as follows:

$$
\begin{aligned}
V(t, X, Y)= & (x(t)-p(t))^{2}+(y(t)-q(t))^{2} \\
& +(u(t)-w(t))^{2}+(v(t)-z(t))^{2} ;
\end{aligned}
$$

it is easy to see that the norm

$$
\begin{aligned}
\|X-Y\|= & |x(t)-p(t)|+|y(t)-q(t)| \\
& +|u(t)-w(t)|+|v(t)-z(t)|
\end{aligned}
$$

and the norm

$$
\begin{aligned}
\|X-Y\|_{*}=\{( & x(t)-p(t))^{2}+(y(t)-q(t))^{2} \\
& \left.+(u(t)-w(t))^{2}+(v(t)-z(t))^{2}\right\}^{1 / 2}
\end{aligned}
$$

are equivalent; that is, there exist two constants $C_{1}>0, C_{2}>$ 0 such that

$$
C_{1}\|X-Y\| \leq\|X-Y\|_{*} \leq C_{2}\|X-Y\|,
$$

thus

$$
\left(C_{1}\|X-Y\|\right)^{2} \leq V(t, X, Y) \leq\left(C_{2}\|X-Y\|\right)^{2} .
$$

Let $a, b \in C\left(\mathbb{R}^{+}, \mathbb{R}^{+}\right), a(x)=C_{1}^{2} x^{2}, b(x)=C_{2}^{2} x^{2}$; thus condition (i) of Lemma 9 is satisfied. In addition,

$$
\begin{aligned}
& \left|V(t, X, Y)-V\left(t, X_{1}, Y_{1}\right)\right| \\
& =\mid(x(t)-p(t))^{2}+(y(t)-q(t))^{2} \\
& +(u(t)-w(t))^{2}+(v(t)-z(t))^{2} \\
& -\left(x_{1}(t)-p_{1}(t)\right)^{2}+\left(y_{1}(t)-q_{1}(t)\right)^{2} \\
& +\left(u_{1}(t)-w_{1}(t)\right)^{2}+\left(v_{1}(t)-z_{1}(t)\right)^{2} \\
& \leq\left|(x(t)-p(t))^{2}-\left(x_{1}(t)-p_{1}(t)\right)^{2}\right| \\
& +\left|(y(t)-q(t))^{2}-\left(y_{1}(t)-q_{1}(t)\right)^{2}\right| \\
& +\left|(u(t)-w(t))^{2}-\left(u_{1}(t)-w_{1}(t)\right)^{2}\right| \\
& +\left|(v(t)-z(t))^{2}-\left(v_{1}(t)-z_{1}(t)\right)^{2}\right| \\
& =\left|(x(t)-p(t))+\left(x_{1}(t)-p_{1}(t)\right)\right| \\
& \times\left|(x(t)-p(t))-\left(x_{1}(t)-p_{1}(t)\right)\right| \\
& +\left|(y(t)-q(t))+\left(y_{1}(t)-q_{1}(t)\right)\right| \\
& \times\left|(y(t)-q(t))-\left(y_{1}(t)-q_{1}(t)\right)\right| \\
& +\left|(u(t)-w(t))+\left(u_{1}(t)-w_{1}(t)\right)\right| \\
& \times\left|(u(t)-w(t))-\left(u_{1}(t)-w_{1}(t)\right)\right| \\
& +\left|(v(t)-z(t))+\left(v_{1}(t)-z_{1}(t)\right)\right| \\
& \times\left|(v(t)-z(t))-\left(v_{1}(t)-z_{1}(t)\right)\right|
\end{aligned}
$$




$$
\begin{aligned}
\leq L\left\{\left(\left|x(t)-x_{1}(t)\right|+\left|y(t)-y_{1}(t)\right|\right.\right. \\
\left.+\left|u(t)-u_{1}(t)\right|+\left|v(t)-v_{1}(t)\right|\right) \\
+\left(\left|p(t)-p_{1}(t)\right|+\left|q(t)-q_{1}(t)\right|\right. \\
\left.\left.\quad+\left|w(t)-w_{1}(t)\right|+\left|z(t)-z_{1}(t)\right|\right)\right\} \\
=L\left\{\left\|X-X_{1}\right\|+\left\|Y-Y_{1}\right\|\right\},
\end{aligned}
$$

where

$$
\begin{gathered}
X_{1}=\left(x_{1}(t), y_{1}(t), u_{1}(t), v_{1}(t)\right), \\
Y_{1}=\left(p_{1}(t), q_{1}(t), w_{1}(t), z_{1}(t)\right), \\
L=4 \max \left\{A_{1}, A_{2}, A_{3}, A_{4}\right\} .
\end{gathered}
$$

Therefore, condition (ii) of Lemma 9 is satisfied. Finally, calculating the $V^{\Delta}(t, X, Y)$ along system (63), we can obtain

$$
\begin{aligned}
V^{\Delta} & (t, X, Y) \\
= & {\left[2(x(t)-p(t))+\mu(t)(x(t)-p(t))^{\Delta}\right](x(t)-p(t))^{\Delta} } \\
& +\left[2(y(t)-q(t))+\mu(t)(y(t)-q(t))^{\Delta}\right](y(t)-q(t))^{\Delta} \\
& +\left[2(u(t)-w(t))+\mu(t)(u(t)-w(t))^{\Delta}\right](u(t)-w(t))^{\Delta} \\
& +\left[2(v(t)-z(t))+\mu(t)(v(t)-z(t))^{\Delta}\right](v(t)-z(t))^{\Delta} .
\end{aligned}
$$

Let

$$
\begin{array}{ll}
\bar{x}(t)=x(t)-p(t), & \bar{y}(t)=y(t)-q(t), \\
\bar{u}(t)=u(t)-w(t), & \bar{v}(t)=v(t)-z(t) .
\end{array}
$$

Then

$$
V(t, X, Y)=\bar{x}^{2}(t)+\bar{y}^{2}(t)+\bar{u}^{2}(t)+\bar{v}^{2}(t)
$$

and (71) is transformed into

$$
\begin{aligned}
V^{\Delta}(t, X, Y)= & {\left[2 \bar{x}(t)+\mu(t) \bar{x}^{\Delta}(t)\right] \bar{x}^{\Delta}(t) } \\
& +\left[2 \bar{y}(t)+\mu(t) \bar{y}^{\Delta}(t)\right] \bar{y}^{\Delta}(t) \\
& +\left[2 \bar{u}(t)+\mu(t) \bar{u}^{\Delta}(t)\right] \bar{u}^{\Delta}(t) \\
& +\left[2 \bar{v}(t)+\mu(t) \bar{v}^{\Delta}(t)\right] \bar{v}^{\Delta}(t) \\
= & V_{1}(t)+V_{2}(t)+V_{3}(t)+V_{4}(t),
\end{aligned}
$$

where $V_{1}(t)=\left[2 \bar{x}(t)+\mu(t) \bar{x}^{\Delta}(t)\right] \bar{x}^{\Delta}(t), V_{2}(t)=[2 \bar{y}(t)+$ $\left.\mu(t) \bar{y}^{\Delta}(t)\right] \bar{y}^{\Delta}(t), V_{3}(t)=\left[2 \bar{u}(t)+\mu(t) \bar{u}^{\Delta}(t)\right] \bar{u}^{\Delta}(t), V_{4}(t)=$ $\left[2 \bar{v}(t)+\mu(t) \bar{v}^{\Delta}(t)\right] \bar{v}^{\Delta}(t)$. Using the mean value theorem, we have

$$
\begin{aligned}
& \exp \{x(t)\}-\exp \{p(t)\}=\xi_{1}(t)(x(t)-p(t)), \\
& \exp \{y(t)\}-\exp \{q(t)\}=\xi_{2}(t)(y(t)-q(t)),
\end{aligned}
$$

where $\xi_{1}(t)$ lies between $\exp \{x(t)\}$ and $\exp \{p(t)\}, \xi_{2}(t)$ lies between $\exp \{y(t)\}$ and $\exp \{q(t)\}$. Substituting (75) into (63), we have

$$
\begin{aligned}
& \bar{x}^{\Delta}(t) \\
& =-a_{1}(t) \xi_{1}(t) \bar{x}(t) \\
& \quad-b_{1}(t)\left[\left(\exp \{y(t)\}+\exp \{q(t)\}-2 c_{2}(t)\right) \xi_{2}(t) \bar{y}(t)\right] \\
& \quad-e_{1}(t) \bar{u}(t), \\
& \bar{y}^{\Delta}(t) \\
& =-a_{2}(t) \xi_{2}(t) \bar{y}(t) \\
& \quad+b_{2}(t)\left[\left(\exp \{x(t)\}+\exp \{p(t)\}-2 c_{1}(t)\right) \xi_{1}(t) \bar{x}(t)\right] \\
& \quad-e_{2}(t) \bar{v}(t), \\
& \quad \bar{u}^{\Delta}(t)=-\alpha_{1}(t) \bar{u}(t)+\beta_{1}(t) \xi_{1}(t) \bar{x}(t), \\
& \quad \bar{v}^{\Delta}(t)=-\alpha_{2}(t) \bar{v}(t)+\beta_{2}(t) \xi_{2}(t) \bar{y}(t),
\end{aligned}
$$

then

$$
\begin{aligned}
& V_{1}(t) \\
& \begin{aligned}
=\{2 \bar{x}(t) \\
+
\end{aligned} \\
& \quad \mu(t) \\
& \quad \times\left(-a_{1}(t) \xi_{1}(t) \bar{x}(t)\right. \\
& \quad-b_{1}(t) \\
& \quad \times\left[\left(\exp \{y(t)\}+\exp \{q(t)\}-2 c_{2}(t)\right) \xi_{2}(t) \bar{y}(t)\right] \\
& \left.\left.\quad-e_{1}(t) \bar{u}(t)\right)\right\} \\
& \times\left(-a_{1}(t) \xi_{1}(t) \bar{x}(t)\right. \\
& \quad-b_{1}(t) \\
& \quad \times\left[\left(\exp \{y(t)\}+\exp \{q(t)\}-2 c_{2}(t)\right) \xi_{2}(t) \bar{y}(t)\right] \\
& \left.\quad-e_{1}(t) \bar{u}(t)\right) \\
& =-2 a_{1}(t) \xi_{1}(t) \bar{x}^{2}(t)+\mu(t) a_{1}^{2}(t) \xi_{1}^{2}(t) \bar{x}^{2}(t) \\
& +\mu(t) b_{1}(t) a_{1}(t) \xi_{1}(t) \xi_{2}(t) \\
& \times\left(\exp \{y(t)\}+\exp \{q(t)\}-2 c_{2}(t)\right) \bar{y}(t) \bar{x}(t) \\
& +\mu(t) a_{1}(t) e_{1}(t) \xi_{1}(t) \bar{x}(t) \bar{u}(t) \\
& -2 b_{1}(t) \xi_{2}(t) \bar{x}(t) \bar{y}(t) \\
& \times\left(\exp \{y(t)\}+\exp \{q(t)\}-2 c_{2}(t)\right) \\
& +\mu(t) b_{1}(t) a_{1}(t) \xi_{1}(t) \xi_{2}(t) \bar{x}(t) \bar{y}(t) \\
& \times\left(\exp \{y(t)\}+\exp \{q(t)\}-2 c_{2}(t)\right)
\end{aligned}
$$




$$
\begin{aligned}
& +\mu(t) b_{1}^{2}(t) \xi_{2}^{2}(t) \bar{y}^{2}(t) \\
& \times\left(\exp \{y(t)\}+\exp \{q(t)\}-2 c_{2}(t)\right)^{2} \\
& +\mu(t) e_{1}(t) b_{1}(t) \xi_{2}(t) \bar{y}(t) \bar{u}(t) \\
& \times\left(\exp \{y(t)\}+\exp \{q(t)\}-2 c_{2}(t)\right) \\
& -2 e_{1}(t) \bar{x}(t) \bar{u}(t)+\mu(t) a_{1}(t) \xi_{1}(t) e_{1}(t) \bar{x}(t) \bar{u}(t) \\
& +\mu(t) e_{1}(t) b_{1}(t) \xi_{2}(t) \bar{y}(t) \bar{u}(t) \\
& \times\left(\exp \{y(t)\}+\exp \{q(t)\}-2 c_{2}(t)\right) \\
& +\mu(t) e_{1}^{2}(t) \bar{u}^{2}(t) \\
& =\left(-2 a_{1}(t) \xi_{1}(t)+\mu(t) a_{1}^{2}(t) \xi_{1}^{2}(t)\right) \bar{x}^{2}(t) \\
& +\mu(t) e_{1}^{2}(t) \bar{u}^{2}(t) \\
& +\mu(t) b_{1}^{2}(t) \xi_{2}^{2}(t) \bar{y}^{2}(t) \\
& \times\left(\exp \{y(t)\}+\exp \{q(t)\}-2 c_{2}(t)\right)^{2} \\
& +\left[2 \mu(t) b_{1}(t) a_{1}(t) \xi_{1}(t) \xi_{2}(t)\right. \\
& \times\left(\exp \{y(t)\}+\exp \{q(t)\}-2 c_{2}(t)\right) \\
& -2 b_{1}(t) \xi_{2}(t) \\
& \left.\times\left(\exp \{y(t)\}+\exp \{q(t)\}-2 c_{2}(t)\right)\right] \bar{x}(t) \bar{y}(t) \\
& +2 \mu(t) b_{1}(t) e_{1}(t) \xi_{2}(t) \\
& \times\left(\exp \{y(t)\}+\exp \{q(t)\}-2 c_{2}(t)\right) \bar{y}(t) \bar{u}(t) \\
& +\left(2 \mu(t) a_{1}(t) \xi_{1}(t) e_{1}(t)-2 e_{1}(t)\right) \bar{x}(t) \bar{u}(t) \\
& \leq\left(\mu(t) a_{1}^{M^{2}} \xi_{1}^{M^{2}}-2 a_{1}^{M} \xi_{1}^{M}\right) \bar{x}^{2}(t)+\mu(t) e_{1}^{M^{2}} \bar{u}^{2}(t) \\
& +\mu(t) b_{1}^{M^{2}} \xi_{2}^{M^{2}}\left(2 e^{y^{*}}-2 c_{2}^{m}\right)^{2} \bar{y}^{2}(t) \\
& +\mid \mu(t) b_{1}^{M} a_{1}^{M} \xi_{1}^{M} \xi_{2}^{M}\left(2 e^{y^{*}}-2 c_{2}^{m}\right) \\
& -b_{1}^{m} \xi_{2}^{m}\left(2 e^{y_{*}}-2 c_{2}^{M}\right) \mid\left(\bar{x}^{2}(t)+\bar{y}^{2}(t)\right) \\
& +\mu(t) e_{1}^{M} b_{1}^{M} \xi_{2}^{M}\left(2 e^{y^{*}}-2 c_{2}^{m}\right) \\
& \times\left(\bar{u}^{2}(t)+\bar{y}^{2}(t)\right) \\
& +\left|\mu(t) a_{1}^{M} \xi_{1}^{M} e_{1}^{M}-e_{1}^{m}\right|\left(\bar{u}^{2}(t)+\bar{x}^{2}(t)\right), \\
& V_{2}(t) \\
& =\{2 \bar{y}(t) \\
& +\mu(t) \\
& \times\left(-a_{2}(t) \xi_{2}(t) \bar{y}(t)\right. \\
& +b_{2}(t)
\end{aligned}
$$$$
\begin{aligned}
\times & {\left[\left(\exp \{x(t)\}+\exp \{p(t)\}-2 c_{1}(t)\right)\right.} \\
& \left.\times \xi_{1}(t) \bar{x}(t)\right] \\
& \left.\left.-e_{2}(t) \bar{v}(t)\right)\right\} \\
\times & \left(-a_{2}(t) \xi_{2}(t) \bar{y}(t)\right. \\
+ & b_{2}(t) \\
\times & {\left[\left(\exp \{x(t)\}+\exp \{p(t)\}-2 c_{1}(t)\right) \xi_{1}(t) \bar{x}(t)\right] } \\
- & \left.e_{2}(t) \bar{v}(t)\right)
\end{aligned}
$$$$
=-2 a_{2}(t) \xi_{2}(t) \bar{y}^{2}(t)
$$$$
+\mu(t) a_{2}^{2}(t) \xi_{2}^{2}(t) \bar{y}^{2}(t)
$$$$
-\mu(t) b_{2}(t) \xi_{1}(t) a_{2}(t) \xi_{2}(t)
$$$$
\times\left(\exp \{x(t)\}+\exp \{p(t)\}-2 c_{1}(t)\right) \bar{y}(t) \bar{x}(t)
$$$$
+\mu(t) a_{2}(t) e_{2}(t) \xi_{2}(t) \bar{y}(t) \bar{v}(t)
$$$$
+2 b_{2}(t) \xi_{1}(t)
$$$$
\times\left(\exp \{x(t)\}+\exp \{p(t)\}-2 c_{1}(t)\right) \bar{y}(t) \bar{x}(t)
$$$$
-\mu(t) a_{2}(t) b_{2}(t) \xi_{2}(t) \xi_{1}(t)
$$$$
\times\left(\exp \{x(t)\}+\exp \{p(t)\}-2 c_{1}(t)\right) \bar{y}(t) \bar{x}(t)
$$$$
+\mu(t) b_{2}^{2}(t) \xi_{1}^{2}(t)
$$$$
\times\left(\exp \{x(t)\}+\exp \{p(t)\}-2 c_{1}(t)\right)^{2} \bar{x}^{2}(t)
$$$$
-\mu(t) e_{2}(t) b_{2}(t) \xi_{1}(t)
$$$$
\times\left(\exp \{x(t)\}+\exp \{p(t)\}-2 c_{1}(t)\right) \bar{v}(t) \bar{x}(t)
$$$$
-2 e_{2}(t) \bar{v}(t) \bar{y}(t)+\mu(t) a_{2}(t) e_{2}(t) \xi_{2}(t) \bar{v}(t) \bar{y}(t)
$$$$
+\mu(t) e_{2}^{2}(t) \bar{v}^{2}(t)
$$$$
-\mu(t) e_{2}(t) b_{2}(t) \xi_{1}(t)
$$$$
\times\left(\exp \{x(t)\}+\exp \{p(t)\}-2 c_{1}(t)\right) \bar{v}(t) \bar{x}(t)
$$$$
=\left(\mu(t) a_{2}^{2}(t) \xi_{2}^{2}(t)-2 a_{2}(t) \xi_{2}(t)\right) \bar{y}^{2}(t)
$$$$
+\left[2 b_{2}(t) \xi_{2}(t)\left(\exp \{x(t)\}+\exp \{p(t)\}-2 c_{1}(t)\right)\right.
$$$$
-2 \mu(t) b_{2}(t) \xi_{1}(t) a_{2}(t) \xi_{2}(t)
$$$$
\left.\times\left(\exp \{x(t)\}+\exp \{p(t)\}-2 c_{1}(t)\right)\right] \bar{x}(t) \bar{y}(t)
$$$$
+\left(2 \mu(t) a_{2}(t) \xi_{2}(t) e_{2}(t)-2 e_{2}(t)\right) \bar{v}(t) \bar{y}(t)
$$$$
+\mu(t) b_{2}^{2}(t) \xi_{1}^{2}(t)
$$$$
\times\left(\exp \{x(t)\}+\exp \{p(t)\}-2 c_{1}(t)\right)^{2} \bar{x}^{2}(t)
$$$$
-2 \mu(t) b_{2}(t) \xi_{1}(t) e_{2}(t)
$$$$
\times\left(\exp \{x(t)\}+\exp \{p(t)\}-2 c_{1}(t)\right) \bar{x}(t) \bar{v}(t)
$$ 


$$
\begin{aligned}
& +\mu(t) e_{2}^{2}(t) \bar{v}^{2}(t) \\
& \leq\left(\mu(t) \alpha_{2}^{M^{2}}-2 \alpha_{2}^{m}\right) \bar{v}^{2}(t)+\mu(t) \beta_{2}^{M^{2}} \xi_{2}^{M^{2}} \bar{y}^{2}(t) \\
& +\left(2 \mu(t) a_{2}(t) \xi_{2}(t) e_{2}(t)-2 e_{2}(t)\right) \bar{y}(t) \bar{v}(t) \\
& \leq\left(\mu(t) a_{2}^{M^{2}} \xi_{2}^{M^{2}}-2 a_{2}^{m} \xi_{2}^{m}\right) \bar{y}^{2}(t) \\
& +\left|\beta_{2}^{M} \xi_{2}^{M}-\mu(t) \beta_{2}^{m} \xi_{2}^{m} \alpha_{2}^{m}\right|\left(\bar{y}^{2}(t)+\bar{v}^{2}(t)\right), \\
& +\mu(t) e_{2}^{M^{2}} \bar{v}^{2}(t)+\mu(t) b_{2}^{M^{2}} \xi_{1}^{M^{2}} \\
& \times\left(2 e^{x^{*}}-2 c_{1}^{m}\right)^{2} \bar{x}^{2}(t) \\
& +\mu(t) b_{2}^{M} e_{2}^{M} \xi_{1}^{M}\left(2 e^{x^{*}}-2 c_{1}^{m}\right)\left(\bar{x}^{2}(t)+\bar{v}^{2}(t)\right) \\
& +\mid b_{2}^{M} \xi_{2}^{M}\left(2 e^{x^{*}}-2 c_{1}^{m}\right) \\
& \xi_{1}^{m}=e^{x_{*}}, \quad \xi_{1}^{M}=e^{x^{*}}, \quad \xi_{2}^{m}=e^{y_{*}}, \quad \xi_{2}^{M}=e^{y^{*}} \\
& -\mu(t) a_{2}^{m} b_{2}^{m} \xi_{1}^{m} \xi_{2}^{m}\left(2 e^{x_{*}}-2 c_{1}^{M}\right) \mid \\
& \times\left(\bar{x}^{2}(t)+\bar{y}^{2}(t)\right) \\
& +\left|\mu(t) a_{2}^{M} \xi_{2}^{M} e_{2}^{M}-e_{2}^{m}\right|\left(\bar{y}^{2}(t)+\bar{v}^{2}(t)\right), \\
& V_{3}(t) \\
& =\left(2 \bar{u}(t)+\mu(t) \bar{u}^{\Delta}(t)\right) \bar{u}^{\Delta}(t) \\
& =\left(2 \bar{u}(t)+\mu(t)\left(-\alpha_{1}(t) \bar{u}(t)+\beta_{1}(t) \xi_{1}(t) \bar{x}(t)\right)\right) \\
& \times\left(-\alpha_{1}(t) \bar{u}(t)+\beta_{1}(t) \xi_{1}(t) \bar{x}(t)\right) \\
& =-2 \alpha_{1}(t) \bar{u}^{2}(t)+\mu(t) \alpha_{1}^{2}(t) \bar{u}^{2}(t) \\
& -\mu(t) \beta_{1}(t) \xi_{1}(t) \alpha_{1}(t) \bar{x}(t) \bar{u}(t) \\
& +2 \beta_{1}(t) \xi_{1}(t) \bar{x}(t) \bar{u}(t) \\
& -\mu(t) \beta_{1}(t) \xi_{1}(t) \alpha_{1}(t) \bar{x}(t) \bar{u}(t) \\
& +\mu(t) \beta_{1}^{2}(t) \xi_{1}^{2}(t) \bar{x}^{2}(t) \\
& =\left(\mu(t) \alpha_{1}^{2}(t)-2 \alpha_{1}(t)\right) \bar{u}^{2}(t) \\
& +\mu(t) \beta_{1}^{2}(t) \xi_{1}^{2}(t) \bar{x}^{2}(t) \\
& +\left(2 \beta_{1}(t) \xi_{1}(t)-2 \mu(t) \beta_{1}(t) \xi_{1}(t) \alpha_{1}(t)\right) \bar{x}(t) \bar{u}(t) \\
& \leq\left(\mu(t) \alpha_{1}^{M^{2}}-2 \alpha_{1}^{m}\right) \bar{u}^{2}(t)+\mu(t) \beta_{1}^{M^{2}} \xi_{1}^{M^{2}} \bar{x}^{2}(t) \\
& +\left|\beta_{1}^{M} \xi_{1}^{M}-\mu(t) \beta_{1}^{m} \xi_{1}^{m} \alpha_{1}^{m}\right|\left(\bar{x}^{2}(t)+\bar{u}^{2}(t)\right), \\
& V_{4}(t) \\
& =\left(2 \bar{v}(t)+\mu(t) \bar{v}^{\Delta}(t)\right) \bar{v}^{\Delta}(t) \\
& =\left(2 \bar{v}(t)+\mu(t)\left(-\alpha_{2}(t) \bar{v}(t)+\beta_{2}(t) \xi_{2}(t) \bar{y}(t)\right)\right) \\
& \times\left(-\alpha_{2}(t) \bar{v}(t)+\beta_{2}(t) \xi_{2}(t) \bar{y}(t)\right) \\
& =\left(\mu(t) \alpha_{2}^{2}(t)-2 \alpha_{2}(t)\right) \bar{v}^{2}(t)+\mu(t) \beta_{2}^{2}(t) \xi_{2}^{2}(t) \bar{y}^{2}(t) \\
& +\left(2 \beta_{2}(t) \xi_{2}(t)-2 \mu(t) \beta_{2}(t) \xi_{2}(t) \alpha_{2}(t)\right) \bar{y}(t) \bar{v}(t) \\
& V^{\Delta}=V_{1}+V_{2}+V_{3}+V_{4} \\
& \leq\left(\mu(t) a_{1}^{M^{2}} \xi_{1}^{M^{2}}-2 a_{1}^{M} \xi_{1}^{M}\right. \\
& +\mid \mu(t) b_{1}^{M} a_{1}^{M} \xi_{1}^{M} \xi_{2}^{M}\left(2 e^{y^{*}}-2 c_{2}^{m}\right) \\
& -b_{1}^{m} \xi_{2}^{m}\left(2 e^{y_{*}}-2 c_{2}^{M}\right) \\
& +\left|\mu(t) a_{1}^{M} \xi_{1}^{M} e_{1}^{M}-e_{1}^{m}\right| \\
& +\mu(t) b_{2}^{M^{2}} \xi_{1}^{M^{2}}\left(2 e^{x^{*}}-2 c_{1}^{m}\right)^{2} \\
& +\mu(t) b_{2}^{M} e_{2}^{M} \xi_{1}^{M}\left(2 e^{x^{*}}-2 c_{1}^{m}\right) \\
& +\mid b_{2}^{M} \xi_{2}^{M}\left(2 e^{x^{*}}-2 c_{1}^{m}\right) \\
& -\mu(t) a_{2}^{m} b_{2}^{m} \xi_{1}^{m} \xi_{2}^{m}\left(2 e^{x_{*}}-2 c_{1}^{M}\right) \mid \\
& +\mu(t) \beta_{1}^{M^{2}} \xi_{1}^{M^{2}} \\
& \left.+\left|\beta_{1}^{M} \xi_{1}^{M}-\mu(t) \beta_{1}^{m} \xi_{1}^{m} \alpha_{1}^{m}\right|\right) \bar{x}^{2}(t) \\
& +\left(\mu(t) b_{1}^{M^{2}} \xi_{2}^{M^{2}}\left(2 e^{y^{*}}-2 c_{2}^{m}\right)^{2}\right. \\
& +\mid \mu(t) b_{1}^{M} a_{1}^{M} \xi_{1}^{M} \xi_{2}^{M}\left(2 e^{y^{*}}-2 c_{2}^{m}\right) \\
& -b_{1}^{m} \xi_{2}^{m}\left(2 e^{y_{*}}-2 c_{2}^{M}\right) \mid \\
& +\mu(t) e_{1}^{M} b_{1}^{M} \xi_{2}^{M}\left(2 e^{y^{*}}-2 c_{2}^{m}\right)
\end{aligned}
$$




$$
\begin{aligned}
& +\mu(t) a_{2}^{M^{2}} \xi_{2}^{M^{2}}-2 a_{2}^{m} \xi_{2}^{m} \\
& +\mid b_{2}^{M} \xi_{2}^{M}\left(2 e^{x^{*}}-2 c_{1}^{m}\right) \\
& -\mu(t) a_{2}^{m} b_{2}^{m} \xi_{1}^{m} \xi_{2}^{m}\left(2 e^{x_{*}}-2 c_{1}^{M}\right) \mid \\
& +\left|\mu(t) a_{2}^{M} \xi_{2}^{M} e_{2}^{M}-e_{2}^{m}\right|+\mu(t) \beta_{2}^{M^{2}} \xi_{2}^{M^{2}} \\
& \left.+\left|\beta_{2}^{M} \xi_{2}^{M}-\mu(t) \beta_{2}^{m} \xi_{2}^{m} \alpha_{2}^{m}\right|\right) \bar{y}^{2}(t) \\
& +\left(\mu(t) e_{1}^{M^{2}}+\mu(t) e_{1}^{M} b_{1}^{M} \xi_{2}^{M}\left(2 e^{y^{*}}-2 c_{2}^{m}\right)\right. \\
& +\left|\mu(t) a_{1}^{M} \xi_{1}^{M} e_{1}^{M}-e_{1}^{m}\right|+\left(\mu(t) \alpha_{1}^{M^{2}}-2 \alpha_{1}^{m}\right) \\
& \left.+\left|\beta_{1}^{M} \xi_{1}^{M}-\mu(t) \beta_{1}^{m} \xi_{1}^{m} \alpha_{1}^{m}\right|\right) \bar{u}^{2}(t) \\
& +\left(\mu(t) e_{2}^{M^{2}}+\mu(t) b_{2}^{M} e_{2}^{M} \xi_{1}^{M}\left(2 e^{x^{*}}-2 c_{1}^{m}\right)\right. \\
& +\left|\mu(t) a_{2}^{M} \xi_{2}^{M} e_{2}^{M}-e_{2}^{m}\right|+\left(\mu(t) \alpha_{2}^{M^{2}}-2 \alpha_{2}^{m}\right) \\
& \left.+\left|\beta_{2}^{M} \xi_{2}^{M}-\mu(t) \beta_{2}^{m} \xi_{2}^{m} \alpha_{2}^{m}\right|\right) \bar{v}^{2}(t) \\
& \leq-\left[\left(P_{1}-Q_{1} \mu(t)\right) \bar{x}^{2}(t)+\left(P_{2}-Q_{2} \mu(t)\right) \bar{y}^{2}(t)\right. \\
& \left.+\left(R_{1}-S_{1} \mu(t)\right) \bar{u}^{2}(t)+\left(R_{2}-S_{2} \mu(t)\right) \bar{v}^{2}(t)\right] \\
& \leq-\left[\left(P_{1}-Q_{1} \mu\right) \bar{x}^{2}(t)+\left(P_{2}-Q_{2} \mu\right) \bar{y}^{2}(t)\right. \\
& \left.+\left(R_{1}-S_{1} \mu\right) \bar{u}^{2}(t)+\left(R_{2}-S_{2} \mu\right) \bar{v}^{2}(t)\right] \\
& \leq-c\left(\bar{x}^{2}(t)+\bar{y}^{2}(t)+\bar{u}^{2}(t)+\bar{v}^{2}(t)\right) \\
& =-c V(t, X, Y) \text {, }
\end{aligned}
$$

where

$$
\begin{aligned}
c= & \min \left\{P_{1}-Q_{1} \mu, P_{2}-Q_{2} \mu, R_{1}-S_{1} \mu, R_{2}-S_{2} \mu\right\}, \\
P_{1}= & 2 a_{1}^{m} \xi_{1}^{m}-b_{1}^{m} \xi_{2}^{m}\left(2 e^{y_{*}}-2 c_{2}^{M}\right)-e_{1}^{m} \\
& -b_{2}^{M} \xi_{2}^{M}\left(2 e^{x^{*}}-2 c_{1}^{m}\right)-\beta_{1}^{M} \xi_{1}^{M}, \\
Q_{1}= & a_{1}^{M^{2}} \xi_{1}^{M^{2}}+b_{1}^{M} a_{1}^{M} \xi_{1}^{M} \xi_{2}^{M}\left[2 e^{y^{*}}-2 c_{2}^{m}\right] \\
& +a_{1}^{M} \xi_{1}^{M} e_{1}^{M}+b_{2}^{M^{2}} \xi_{1}^{M^{2}}\left[2 e^{x^{*}}-2 c_{1}^{m}\right]^{2} \\
& +b_{2}^{M} e_{2}^{M} \xi_{1}^{M}\left[2 e^{x^{*}}-2 c_{1}^{m}\right]+\beta_{1}^{M^{2}} \xi_{2}^{M^{2}} \\
& +a_{2}^{m} b_{2}^{m} \xi_{1}^{m} \xi_{2}^{m}\left[2 e^{x^{*}}-2 c_{1}^{M}\right]+\beta_{1}^{m} \xi_{1}^{m} \alpha_{1}^{m}, \\
P_{2}= & 2 a_{2}^{m} \xi_{2}^{m}-b_{1}^{m} \xi_{2}^{m}\left(2 e^{y_{*}}-2 c_{2}^{M}\right)-e_{2}^{m} \\
& -b_{2}^{M} \xi_{2}^{M}\left(2 e^{x^{*}}-2 c_{1}^{m}\right)-\beta_{2}^{M} \xi_{2}^{M},
\end{aligned}
$$

$$
\begin{aligned}
Q_{2}= & a_{2}^{M^{2}} \xi_{2}^{M^{2}}+b_{1}^{M} a_{1}^{M} \xi_{1}^{M} \xi_{2}^{M}\left[2 e^{y^{*}}-2 c_{2}^{m}\right] \\
& +b_{1}^{M^{2}} \xi_{2}^{M^{2}}\left[2 e^{y^{*}}-2 c_{2}^{m}\right]^{2}+b_{1}^{M} e_{1}^{M} \xi_{2}^{M}\left[2 e^{y^{*}}-2 c_{2}^{m}\right] \\
& +a_{2}^{m} \xi_{2}^{m} b_{2}^{m} \xi_{1}^{m}\left[2 e^{x_{*}}-2 c_{1}^{M}\right]+a_{2}^{M} \xi_{2}^{M} e_{2}^{M} \\
& +\beta_{2}^{M^{2}} \xi_{2}^{M^{2}}+\beta_{2}^{m} \xi_{2}^{m} \alpha_{2}^{m}, \\
R_{i}= & 2 \alpha_{i}^{m}-\beta_{i}^{M} \xi_{i}^{M}-e_{i}^{m}, \quad(i=1,2) \\
S_{1}= & e_{1}^{M^{2}}+e_{1}^{M} b_{1}^{M} \xi_{2}^{M}\left(2 e^{y^{*}}-2 c_{2}^{m}\right) \\
& +a_{1}^{M} \xi_{1}^{M} e_{1}^{M}+\alpha_{1}^{M^{2}}+\beta_{1}^{m} \xi_{1}^{m} \alpha_{1}^{m} \\
S_{2}= & e_{2}^{M^{2}}+e_{2}^{M} b_{2}^{M} \xi_{1}^{M}\left(2 e^{x^{*}}-2 c_{1}^{m}\right) \\
& +a_{2}^{M} \xi_{2}^{M} e_{2}^{M}+\alpha_{2}^{M^{2}}+\beta_{2}^{m} \xi_{2}^{m} \alpha_{2}^{m} .
\end{aligned}
$$

From the condition $\left(\mathrm{H}_{3}\right), c>0$ and $-c \in \mathscr{R}^{+}$, we see that the condition (iii) of Lemma 9 is satisfied. So all the conditions of Lemma 9 are satisfied. By Lemma 9, there exists a unique uniformly asymptotically stable almost periodic solution $X(t)=(x(t), y(t), u(t), v(t))^{T}$ of system (3) which satisfies

$$
\begin{array}{ll}
x_{*} \leq x(t) \leq x^{*}, & y_{*} \leq y(t) \leq y^{*}, \\
u_{*} \leq u(t) \leq u^{*}, & v_{*} \leq v(t) \leq v^{*}
\end{array}
$$

and $X(t) \in \Omega$ for all $t \in \mathbb{T}$. This completes the proof.

Corollary 16. Assume that $\left(H_{1}\right)-\left(H_{3}\right)$ hold. Suppose that the nonnegative coefficients $r_{i}(t), a_{i}(t), b_{i}(t), c_{i}(t), e_{i}(t), \alpha_{i}(t), \beta_{i}(t)$, $i=1,2$ are periodic of period $\omega$; then system (3) has a unique uniformly asymptotically stable periodic solution of period $\omega$.

\section{An Example}

In this section, we present an example to illustrate the feasibility and effectiveness of our results.

Consider the system (3) with the following coefficients

$$
\begin{aligned}
& r_{1}(t)=14.5+0.5 \sin t+0.5 \cos \sqrt{2} t, \\
& r_{2}(t)=14.5+0.4 \cos t+0.6 \sin \sqrt{2} t, \\
& a_{1}(t)=10.5+0.2 \sin t+0.3 \cos \pi t, \\
& a_{2}(t)=10.5+0.4 \cos \sqrt{2} t+0.1 \cos t, \\
& b_{1}(t)=0.3+0.1 \sin \sqrt{2} t+0.1 \sin \sqrt{3} t, \\
& b_{2}(t)=0.3+0.1 \cos t+0.1 \sin \sqrt{2} t,
\end{aligned}
$$




$$
\begin{aligned}
& c_{1}(t)=1.05+0.02 \sin 2 t+0.03 \cos \sqrt{2} t \\
& c_{2}(t)=1.05+0.03 \sin 2 t+0.02 \cos \sqrt{2} t \\
& \alpha_{1}(t)=1.1+0.05 \sin t+0.05 \cos \sqrt{2} t \\
& \alpha_{2}(t)=1.1+0.03 \sin t+0.07 \cos \sqrt{2} t \\
& \beta_{1}(t)=0.5+0.1 \cos \sqrt{2} t \\
& \beta_{2}(t)=0.5+0.1 \sin \sqrt{3} t \\
& e_{1}(t)=0.5+0.4 \cos \sqrt{2} t \\
& e_{2}(t)=0.5+0.4 \sin \sqrt{3} t
\end{aligned}
$$

Let $\mathbb{T}=\bigcup_{k=0}^{\infty}[k, k+0.75]$. Then system (3) is persistent and has a unique uniformly asymptotically stable almost periodic solution.

Proof. By calculation, we have that

$$
\begin{aligned}
& \mu(t)= \begin{cases}0, & t \in \bigcup_{k=0}^{\infty}[k, k+0.75), \\
0.25, & t \in \bigcup_{k=0}^{\infty}\{k+0.75\},\end{cases} \\
& x^{*}=0.5, \quad y^{*} \approx 0.63591, \\
& u^{*} \approx 0.9892, \quad v^{*} \approx 1.1332, \\
& x_{*} \approx 0.0961, \quad y_{*} \approx 0.1195 \text {, } \\
& u_{*} \approx 0.3670, \quad v_{*} \approx 0.3756 \text {, } \\
& c \approx 1.0478, \quad \mu=0.25 .
\end{aligned}
$$

So all conditions are satisfied and the results follow from Theorems 13 and 15. Therefore, system (3) is persistent and has a unique uniformly asymptotically stable almost periodic solution. This completes the proof.

\section{Acknowledgments}

This work is supported by the National Natural Sciences Foundation of People's Republic of China under Grant 11361072 and the YNU Postdoctoral Science Foundation Project under Grant W4030002.

\section{References}

[1] H. Zhou, "Enterprise cluster co-existence model and stability analysis," Systems Engineering, vol. 21, no. 4, pp. 32-37, 2003 (Chinese).

[2] W. H. Li and F. R. Han, "Studies on coordinated evlution laws between industry populations and imperial analysis," Chinese Journal of Management Science, vol. 12, no. 5, pp. 137-143, 2004 (Chinese).
[3] Q. Guo, "Competitive strategies in an enterprise: an ecological model," Contemporary Economic Management, vol. 27, no. 2, pp. 49-52, 2005 (Chinese).

[4] B. T. Wang and H. Y. Shu, "Research on competition in mobile communications market: ecological model," Journal of Beijing Union University (Natural Sciences), vol. 20, no. 2, pp. 27-30, 2006 (Chinese).

[5] X. H. Tian and Q. K. Nie, "On model construction of enterprises' interactive relationship from the perspective of business ecosystem," Southern Economic Journal, vol. 4, pp. 50-57, 2006 (Chinese).

[6] Z. Wang and R. Y. Pan, "Analysis to the equilibrium of industrial clusters based on ecological model," Statistical Thinktank, vol. 8, pp. 32-35, 2008 (Chinese).

[7] R. Zhang, X. S. Qian, and Z. Gao, "A competitive model of enterprises based on ecology theory," Systems Engineering, vol. 26, pp. 116-119, 2008 (Chinese).

[8] Y. Li and T. Zhang, "Global asymptotical stability of a unique almost periodic solution for enterprise clusters based on ecology theory with time-varying delays and feedback controls," Communications in Nonlinear Science and Numerical Simulation, vol. 17, no. 2, pp. 904-913, 2012.

[9] P. Liu and Y. Li, "Permanence for a competition and cooperation model of enterprise cluster with delays and feedback controls," Electronic Journal of Differential Equations, vol. 2013, no. 22, pp. $1-9,2013$

[10] M. E. Porter, "Cluster and the new economics of competition," Harvard Business Review, vol. 76, no. 6, pp. 77-90, 1998.

[11] J. C. Wang, The Space of Innovation-Enterprise Cluster and Region Development, Beijing University Press, Beijing, China, 2001.

[12] H. Y. Yi and W. Liu, "Identifying the core competitive power of company based on the model of product population competition," Journal of Management Sciences, vol. 20, no. 3, pp. 16-22, 2007 (Chinese).

[13] R. Zhang, X. S. Qian, and Z. Gao, "competitive model of enterprises based on ecology theory," Systems Engineering, vol. 26, pp. 116-119, 2008 (Chinese).

[14] P. Liu and Y. Li, "Multiple positive periodic solutions of nonlinear functional differential system with feedback control," Journal of Mathematical Analysis and Applications, vol. 288, no. 2, pp. 819-832, 2003.

[15] H.-F. Huo and W.-T. Li, "Positive periodic solutions of a class of delay differential system with feedback control," Applied Mathematics and Computation, vol. 148, no. 1, pp. 35-46, 2004.

[16] X. Liao, Z. Ouyang, and S. Zhou, "Permanence of species in nonautonomous discrete Lotka-Volterra competitive system with delays and feedback controls," Journal of Computational and Applied Mathematics, vol. 211, no. 1, pp. 1-10, 2008.

[17] T. Zhang, Y. Li, and Y. Ye, "Persistence and almost periodic solutions for a discrete fishing model with feedback control," Communications in Nonlinear Science and Numerical Simulation, vol. 16, no. 3, pp. 1564-1573, 2011.

[18] A. M. Fink, Almost Periodic Differential Equations, vol. 377 of Lecture Notes in Mathematics, Springer, Berlin, Germany, 1974.

[19] C. Y. He, Almost Periodic Differential Equation, Higher Eduction Publishing House, Beijing, China, 1992.

[20] Y. Li and X. Fan, "Existence and globally exponential stability of almost periodic solution for Cohen-Grossberg BAM neural networks with variable coefficients," Applied Mathematical Modelling, vol. 33, no. 4, pp. 2114-2120, 2009. 
[21] Y. K. Li, T. Y. Zhang, and Z. W. Xing, "The existence of nonzero almost periodic solution for Cohen-Grossberg neural networks with continuously distrbuted delays and impulses," Neurocomputing, vol. 73, pp. 3105-3113, 2010.

[22] K. Wang and Y. L. Zhu, "Stability of almost periodic solution for a generalized neutral-type neural networks with delays," Neurocomputing, vol. 73, pp. 3300-3307, 2010.

[23] J. Geng and Y. Xia, "Almost periodic solutions of a nonlinear ecological model," Communications in Nonlinear Science and Numerical Simulation, vol. 16, no. 6, pp. 2575-2597, 2011.

[24] J. O. Alzabut, G. T. Stamov, and E. Sermutlu, "Positive almost periodic solutions for a delay logarithmic population model," Mathematical and Computer Modelling, vol. 53, no. 1-2, pp. 161167, 2011.

[25] M. Bohner and A. Peterson, Dynamic Equations on Time Scales: An Introduction with Applications, Birkhäuser, Boston, Mass, USA, 2001.

[26] M. Bohner and A. Peterson, Advances in Dynamic Equations on Time Scales, Birkhäuser, Boston, Mass, USA, 2003.

[27] Y. K. Li and C. Wang, "Uniformly almost periodic functions and almost periodic solutions to dynamic equations on time scales," Abstract and Applied Analysis, vol. 2011, Article ID 341520, 22 pages, 2011.

[28] Y. K. Li and X. F. Han, "Almost periodic solution for a N-species competition model with feedback controls on time scales," Journal of Applied Mathematics \& Informatics, vol. 31, pp. 247262, 2013.

[29] Y. K. Li, L. Yang, and H. T. Zhang, "Permanence anduniformly asymptotical stability of almost periodic solutions for a singlespecies model with feedback control on time scales," AsianEuropean Journal of Mathematics. In press. 


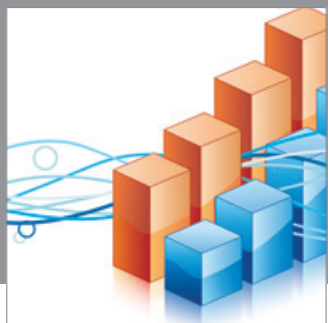

Advances in

Operations Research

mansans

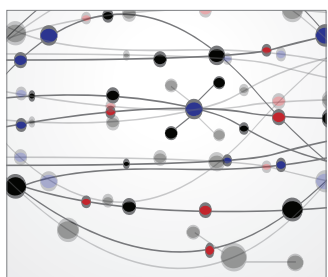

The Scientific World Journal
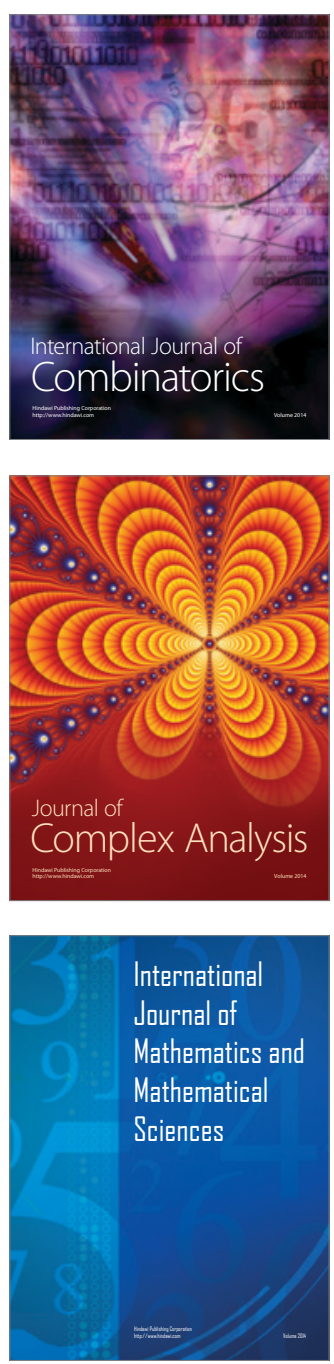
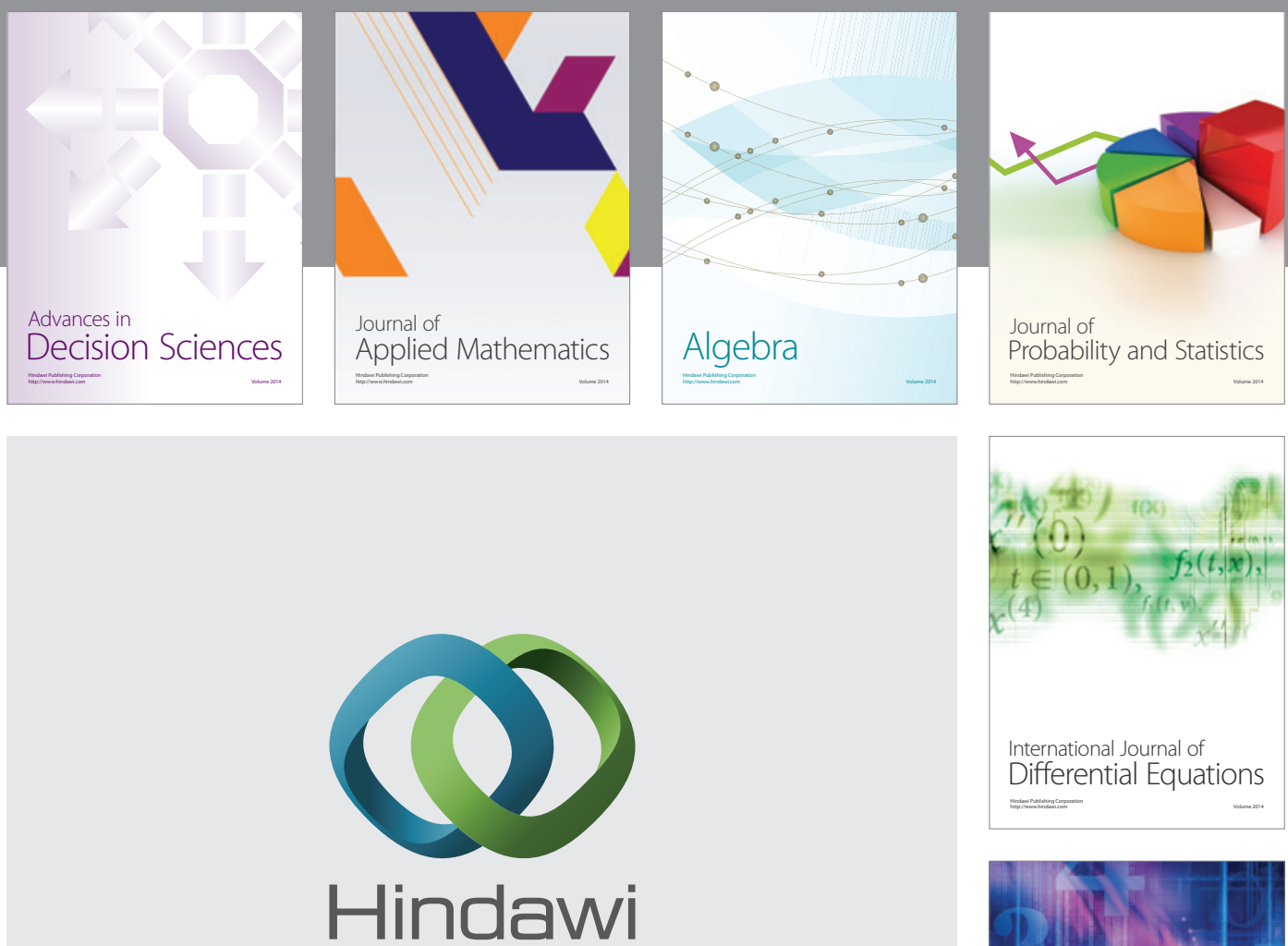

Submit your manuscripts at http://www.hindawi.com
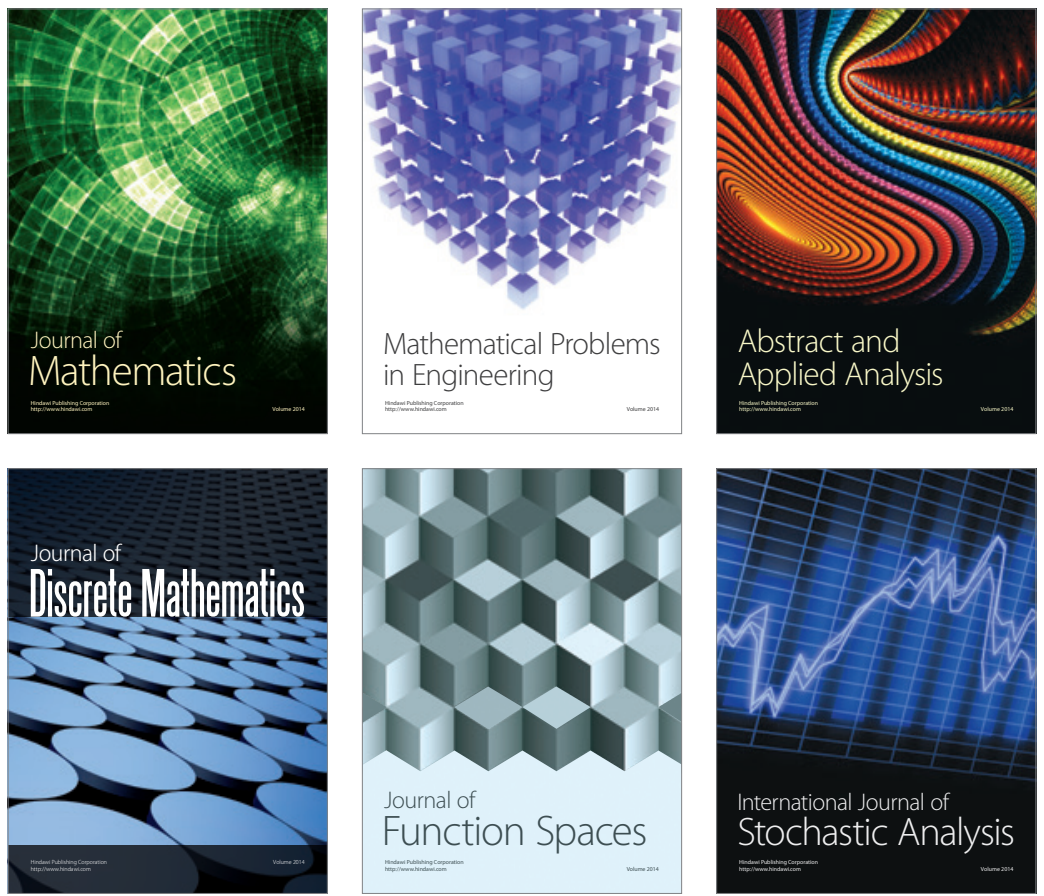

Journal of

Function Spaces

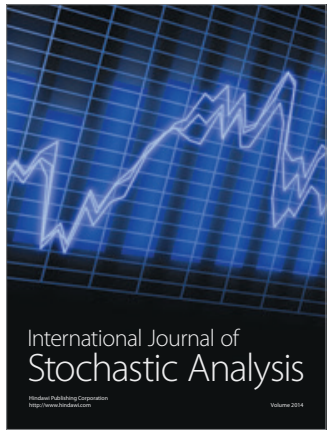

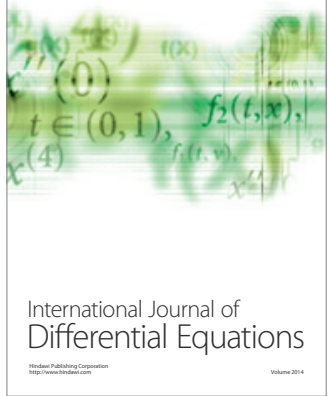
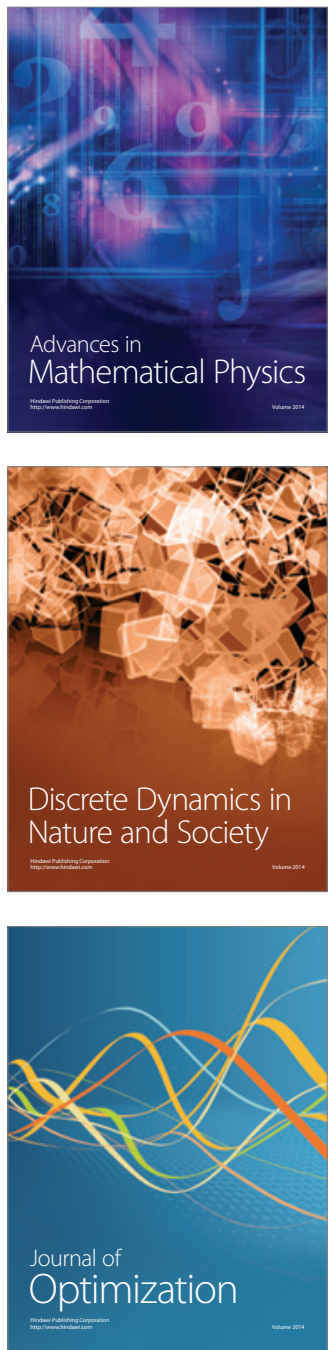